\title{
Thalamic and Entorhinal Network Activity Differently Modulates the Functional Development of Prefrontal-Hippocampal Interactions
}

\author{
Henrike Hartung, ${ }^{1 \star}$ Marco D. Brockmann, ${ }^{1 \star}$ Beatrice Pöschel, ${ }^{1}$ Vito De Feo, ${ }^{1,2}$ and Ileana L. Hanganu-Opatz ${ }^{1}$ \\ ${ }^{1}$ Developmental Neurophysiology, Institute of Neuroanatomy, University Medical Center Hamburg-Eppendorf, 20251 Hamburg, Germany, and \\ ${ }^{2}$ Laboratory of Neural Computation, Center for Neuroscience and Cognitive Systems, Istituto Italiano di Tecnologia, 38068 Rovereto, Italy
}

Precise information flow during mnemonic and executive tasks requires the coactivation of adult prefrontal and hippocampal networks in oscillatory rhythms. This interplay emerges early in life, most likely as an anticipatory template of later cognitive performance. At neonatal age, hippocampal theta bursts drive the generation of prefrontal theta-gamma oscillations. In the absence of direct reciprocal interactions, the question arises of which feedback mechanisms control the early entrainment of prefrontal-hippocampal networks. Here, we demonstrate that prefrontal-hippocampal activity couples with discontinuous theta oscillations and neuronal firing in both lateral entorhinal cortex and ventral midline thalamic nuclei of neonatal rats. However, these two brain areas have different contributions to the neonatal long-range communication. The entorhinal cortex mainly modulates the hippocampal activity via direct axonal projections. In contrast, thalamic theta bursts are controlled by the prefrontal cortex via mutual projections and contribute to hippocampal activity. Thus, the neonatal prefrontal cortex modulates the level of hippocampal activation by directed interactions with the ventral midline thalamus. Similar to the adult task-related communication, theta-band activity ensures the feedback control of long-range coupling in the developing brain.

Key words: development; directed interactions; networks; oscillations; synchrony

\section{Significance Statement}

Memories are encoded by finely tuned interactions within large-scale neuronal networks. This cognitive performance is not inherited, but progressively matures in relationship with the establishment of long-range coupling in the immature brain. The hippocampus initiates and unidirectionally drives the oscillatory entrainment of neonatal prefrontal cortex, yet feedback interactions that precisely control this early communication are still unresolved. Here, we identified distinct roles of entorhinal cortex and ventral midline thalamus for the functional development of prefrontal-hippocampal interactions. While entorhinal oscillations modulate the hippocampal activity by timing the neuronal firing via monosynaptic afferents, thalamic nuclei act as a relay station routing prefrontal activation back to hippocampus. Understanding the mechanisms of network maturation represents the prerequisite for assessing circuit dysfunction in neurodevelopmental disorders.

\section{Introduction}

Long-range communication between remote brain areas enables sensory perception and cognitive processing (Schnitzler and

Received Aug. 28, 2015; revised Jan. 14, 2016; accepted Jan. 21, 2016.

Author contributions: I.L.H.-O. designed research; H.H., M.D.B., B.P., and I.L.H.-O. performed research; H.H., M.D.B., B.P., V.D.F., and I.L.H.-0. analyzed data; H.H., M.D.B., V.D.F., and I.L.H.-0. wrote the paper.

I.L.H.-0. acknowledges support by the Emmy Noether-Program (Grant Ha4466/3-1), the Priority Program 1665 (Grant Ha4466/7-1), and Sonderforschungsbereich Grant 936 (B5) from the German Research Foundation, and the German Federal Ministry of Education and Research (Grant 01G00809). We thank Annette Marquardt and Achim Dahlmann for excellent technical assistance.

*H.H. and M.D.B. contributed equally as first authors.

Correspondence should be addressed to lleana L. Hanganu-Opatz, Developmental Neurophysiology, Institute of Neuroanatomy, University Medical Center Hamburg-Eppendorf, Falkrenried 94, 20251 Hamburg, Germany. E-mail: hangop@zmnh.uni-hamburg.de or ileana.hanganu-opatz@zmnh.uni-hamburg.de.
Gross, 2005). The mutual exchange of information takes place either along direct axonal pathways or via specific relay stations and is precisely controlled by feedback interactions. Such information processing occurs within computation windows of network oscillations (Buzsáki, 2010), as exemplarily illustrated in the case of functional interplay between prefrontal cortex (PFC) and hippocampus (HP; Colgin, 2011). Simultaneous recordings from both regions demonstrated that hippocampal theta-band activity

H. Hartung's present address: Laboratory of Neurobiology, Department of Biosciences, University of Helsinki, FIN-00014 Helsinki, Finland.

M.D. Brockmann's present address: Center for Memory and Brain, Boston University, Boston, MA 02215.

DOI:10.1523/JNEUROSCI.3232-15.2016

Copyright $\odot 2016$ the authors $\quad 0270-6474 / 16 / 363676-15 \$ 15.00 / 0$ 
modulates the firing of prefrontal neurons (Siapas and Wilson, 1998). The resulting temporal coordination, which relies on strong monosynaptic glutamatergic inputs from the CA1 area and subiculum of HP to PFC (Swanson, 1981; Jay and Witter, 1991), is behaviorally relevant for memory encoding (Spellman et al., 2015).

In the absence of direct projections from PFC back to HP, several brain areas have been proposed to precisely control prefrontal-hippocampal coupling. Especially the nucleus reuniens (RE) of ventral midline thalamus (VMT) as well as the entorhinal cortex with its lateral (LEC) and medial (MEC) subdivisions may act as such nodal hubs. Dense reciprocal afferents and efferents (Hoover and Vertes, 2012), as well as dual collaterals to PFC and HP of neurons in these thalamic nuclei (Varela et al., 2014) represent the anatomical substrate of the functional relay by which nuclei of VMT coordinate the prefrontal and hippocampal activity during cognitive tasks (Hembrook et al., 2012). In line with this, electrical stimulation of RE activated the pyramidal CA1 neurons and modulated the prelimbic and infralimbic activity (Eleore et al., 2011). Most of RE neurons showed an increased rate of discharge during theta-band network oscillations (Morales et al., 2007). On the other hand, especially LEC is reciprocally linked with PFC and HP (Jones and Witter, 2007), and this connectivity might underlie the theta-band entorhinalhippocampal communication (Igarashi et al., 2014). At a behavioral level, this communication has been hypothesized to modulate learning and the expression of acquired memory (Takehara-Nishiuchi et al., 2011; Morrissey et al., 2012).

While the directed interplay within prefrontal-hippocampal networks has been extensively investigated in adults, its ontogeny is less well elucidated (Mohns and Blumberg, 2010). We recently showed that monosynaptic projections from pyramidal CA1 neurons drive the initial entrainment in gamma oscillations of local neuronal networks in the prefrontal subdivision prelimbic cortex (PL; Brockmann et al., 2011; Bitzenhofer and Hanganu-Opatz, 2014; Bitzenhofer et al., 2015). Without being immediately involved in overt behavior, these neonatal directed interactions seem to control the emergence of recognition memory at a juvenile age (Krüger et al., 2012). However, an optimal communication within prefrontalhippocampal networks requires that PL equally controls the strength of the hippocampal drive. In the absence of direct feedback (Brockmann et al., 2011), it can be hypothesized that, similarly to the interactions in adults, LEC or the nuclei of VMT act as relay stations tuning the reciprocal influence of PL on hippocampal activity. The contribution of entorhinal and thalamic activity to network wiring during neonatal development is poorly elucidated. Solely, the ontogeny of neural representation of space has recently been assessed in MEC (Langston et al., 2010; Wills et al., 2010, 2012). While the earliest developing spatial responses in this area, the headdirection cells, emerge during the second postnatal week (Bjerknes et al., 2015; Tan et al., 2015), in vitro studies identified already around birth organized firing of neurons in MEC that might contribute to entorhinal tuning and functional clustering (Dawitz et al., 2012).

To decide whether activation of LEC and/or VMT facilitates reciprocal communication and feedback control within neonatal prefrontal-hippocampal networks, we combined multisite recordings of multiunit activity (MUA) and local field potentials (LFPs) in vivo with anatomical tracing and reversible inactivation of neuronal activity in rats at the developmental stage of maximal drive from $\mathrm{HP}$ to PL (i.e., end of the first postnatal week). We show that, in line with the directed oscillatory coupling and spike-timing relationships, LEC and VMT have distinct functions for early information transfer within prefrontal-hippocampal networks during neonatal development.

\section{Materials and Methods}

\section{Surgical preparation}

All experiments were performed in compliance with German laws and the guidelines of the European Community for the use of animals in research, and were approved by the local ethical committee. Pregnant Wistar rats were obtained at $14-17 \mathrm{~d}$ of gestation from the animal facility of the University Medical Center Hamburg-Eppendorf, were housed individually in breeding cages with a $12 \mathrm{~h}$ light/dark cycle, and were fed ad libitum. Extracellular recordings were performed in PFC $(1.5-1.8 \mathrm{~mm}$ anterior to bregma and $0.2-0.5 \mathrm{~mm}$ from the midline) and intermediate HP (iHP; $4.5 \mathrm{~mm}$ posterior to bregma and $4.5 \mathrm{~mm}$ from the midline), LEC ( $5.5 \mathrm{~mm}$ posterior to bregma and $7.5 \mathrm{~mm}$ from the midline), and VMT ( $1.5 \mathrm{~mm}$ posterior to bregma and $0.1 \mathrm{~mm}$ from the midline) of postnatal day (P) 7 to $\mathrm{P} 9$ male rats using experimental protocols as described previously (Brockmann et al., 2011). Under light urethaneanesthesia ( $1 \mathrm{~g} / \mathrm{kg}$; Sigma-Aldrich), the head of the pup was fixed into the stereotaxic apparatus (Stoelting) using two metal bars fixed with dental cement on the nasal and occipital bones, respectively. The bone over the regions of interest was carefully removed by drilling holes $<0.5 \mathrm{~mm}$ in diameter. Removal of the underlying dura mater by drilling was avoided, since leakage of CSF or blood damps the cortical activity and single neuronal firing (I. Hanganu-Opatz, unpublished observations). The body of the animal was surrounded by cotton and kept at a constant temperature of $37^{\circ} \mathrm{C}$ by placing it on a heating blanket. After a $20-30 \mathrm{~min}$ recovery period, multielectrode arrays (Silicon Michigan probes, NeuroNexus Technologies) were inserted perpendicularly into the skull surface into PFC to a depth of $3 \mathrm{~mm}$, at $20^{\circ}$ from the vertical plane into iHP at a depth of $1.8-2.2 \mathrm{~mm}$, at $10^{\circ}$ from the vertical plane into LEC to a depth of $4.5 \mathrm{~mm}$, and perpendicularly into VMT to a depth of $5.5 \mathrm{~mm}$. The electrodes were labeled with DiI (1,1'-dioctadecyl-3,3,3',3'tetramethylindocarbocyanine, Invitrogen) to enable postmortem in histological sections the reconstruction of their tracks in LEC and VMT (Fig. $1 A, E)$ as well as in PFC and iHP. Three silver wires were inserted into the cerebellum, and served as ground and reference electrodes.

\section{Recording protocols}

Simultaneous recordings of LFP and MUA were performed from PFC, iHP, and LEC or from PFC, iHP, and VMT using one-shank 8- or 16-channel Michigan electrodes $(0.5-3 \mathrm{M} \Omega$ ). The recording sites were separated by 50 , 100 , or $200 \mu \mathrm{m}$ in vertical direction. According to postmortem histological evaluation, the recording sites covered layers II/III of the prefrontal subdivisions cingulate and PL (Van Eden and Uylings, 1985), the stratum pyramidale of CA1 area in the iHP, layers II/III of LEC, as well as the nuclei RE and rhomboid of VMT. Both LFP and MUA were recorded for at least $900 \mathrm{~s}$ at a sampling rate of 32 or $25 \mathrm{kHz}$ using a multichannel extracellular amplifier (Digital Lynx 4S with no gain, Neuralynx; or USB-ME16-FAI-System with a gain of 1000, Multichannel Systems) and the corresponding acquisition software (Cheetah or MC-RACK). During recordings, the signal was bandpass filtered between $0.1 \mathrm{~Hz}$ and $5 \mathrm{kHz}$ (Digital Lynx), and between $1 \mathrm{~Hz}$ and 5 $\mathrm{kHz}$ (MCS).

\section{Impairment of prefrontal or thalamic activity}

Acute and reversible impairment of prefrontal or thalamic activity was induced by injection of lidocaine (Sigma-Aldrich; $10 \%$ in $0.9 \%$ saline, pH 7.0 with $\mathrm{NaOH}, 300 \mathrm{nl}, 200 \mathrm{nl} / \mathrm{min}$ ) into PL or VMT (100 nl, 100 $\mathrm{nl} / \mathrm{min}$ ) of P7-P8 male rats. The lidocaine acted on a small tissue volume within an estimated radius of $\sim 300-400 \mu \mathrm{m}$ (Tehovnik and Sommer, 1997), and consequently the lidocaine-induced impairment of electrical activity was confined to PL or VMT. Due to the large distances between the silenced area and the other investigated regions (on anterior-posterior axis PL-VMT, 3.5-4 mm; PL-iHP, $6 \mathrm{~mm}$; PL-LEC, 7-8 mm; VMT-iHP, 2-2.5 mm), a direct inactivation of the latter ones by unspecific spread of lidocaine can be excluded. 

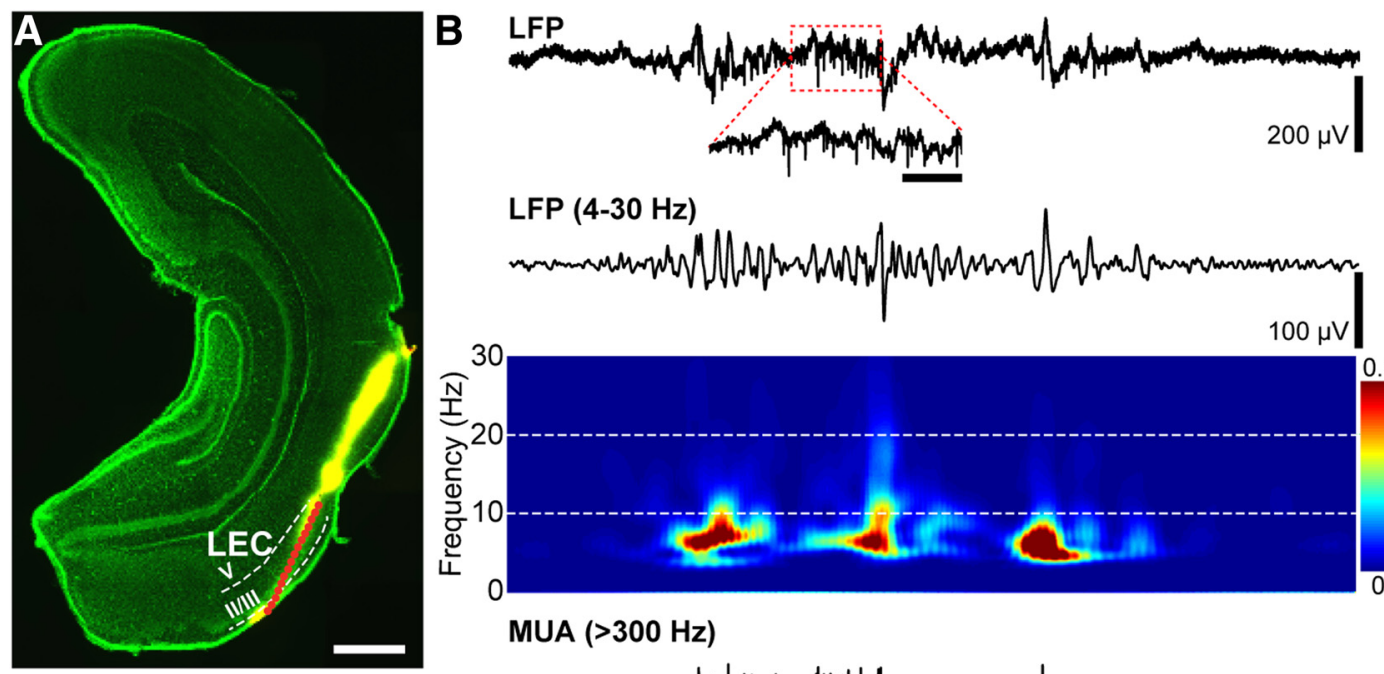

LFP (4-30 Hz)

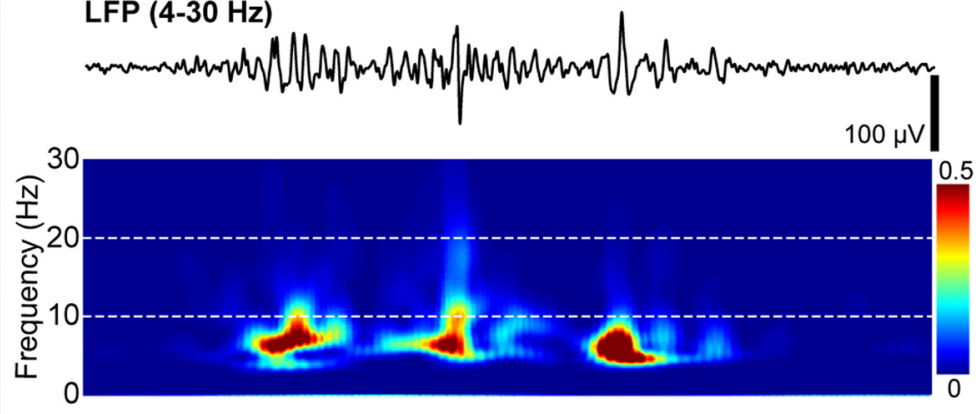

MUA (>300 Hz)

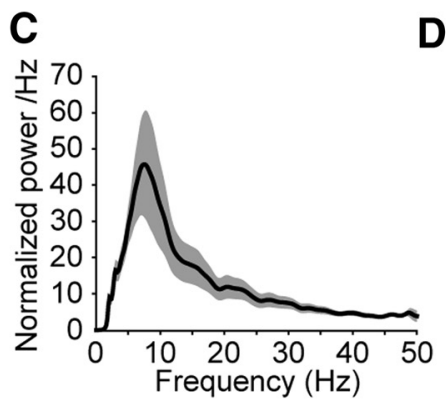

D (i)

(ii)

$100 \mu \mathrm{V}$
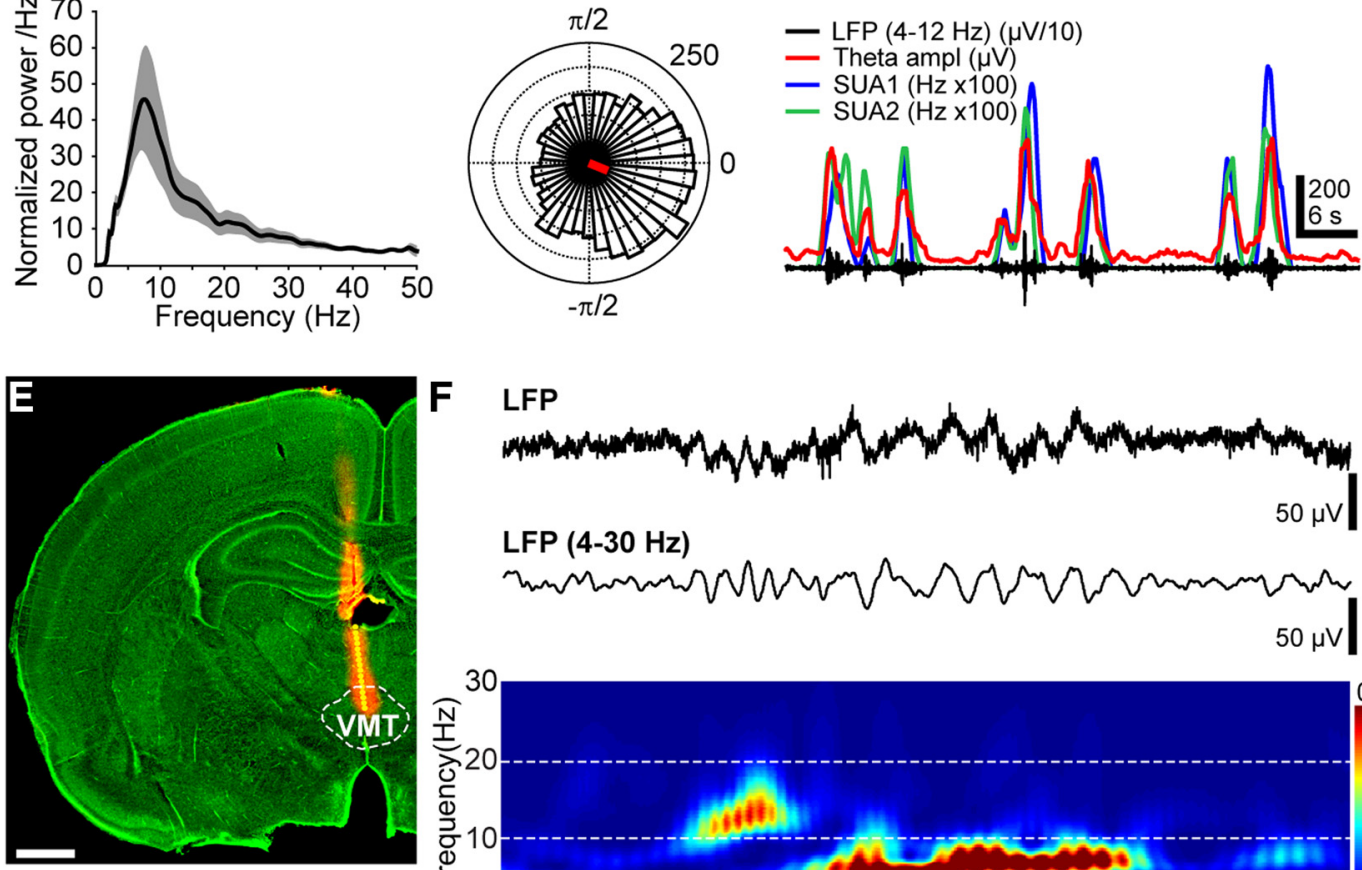

F LFP

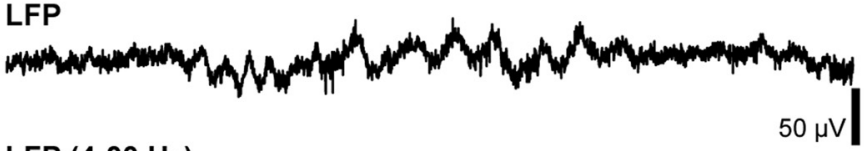

LFP (4-30 Hz)

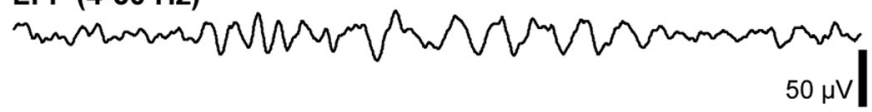

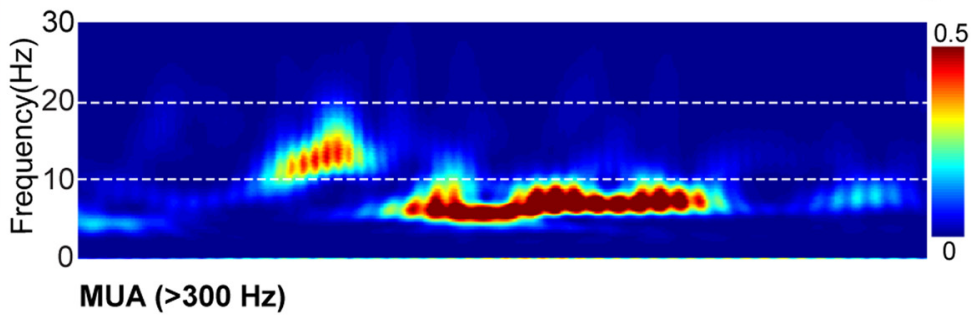

$50 \mu \mathrm{V}$

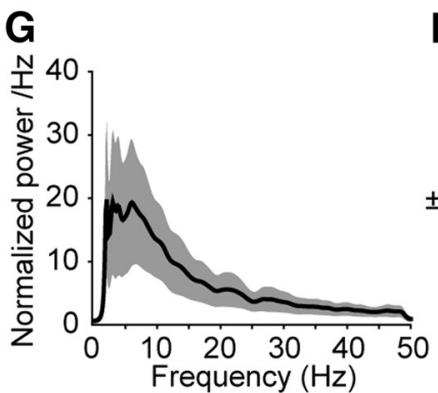

H (i)

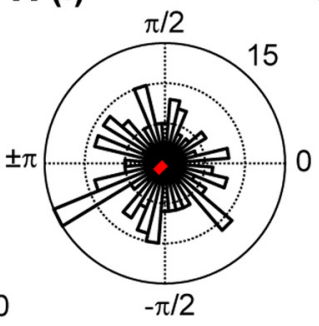

(ii)

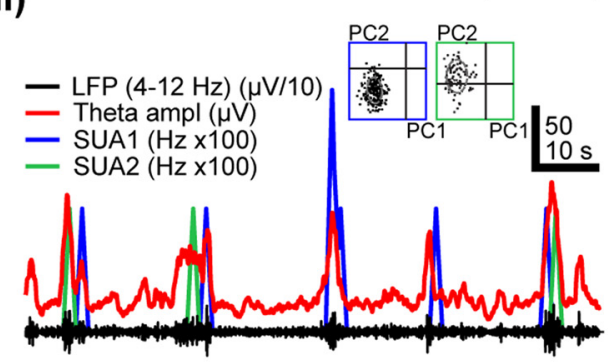

Figure 1. Patterns of network activity in the rat LEC and VMT in vivo at the end of the first postnatal week. $\boldsymbol{A}$, Digital photomontage reconstructing the location of the Dil-labeled recording electrode (yellow) in LEC of a Nissl-stained 100- $\mu \mathrm{m}$-thick coronal section from a P7 rat. The superimposed red dots mark the 16 recording sites covering the entorhinal layers II-III. $\boldsymbol{B}$, Characteristic theta burst from LEC displayed before (top) and after (4-30 Hz, middle) bandpass filtering, and the corresponding MUA after $300 \mathrm{~Hz}$ high-pass filtering (bottom). (Figure legend continues.) 


\section{Retrograde tracing and staining protocols}

Male Wistar rats received at P1 or P5 unilateral and bilateral injections of Fluoro-Gold (FG; Fluorochrome). A total volume of $0.1-0.2 \mu \mathrm{l}$ of FG (2.5\% in PBS) was delivered via a $10 \mu \mathrm{l}$ microsyringe pump controller into PFC. The slow injection speed $(0.1 \mu \mathrm{l} / \mathrm{min})$ and the maintenance of the syringe in place for at least 3 min ensured an optimal diffusion of the tracer. After a survival time of 3-6 d, the rats were deeply anesthetized with $10 \%$ ketamine (aniMedica) and $2 \%$ xylazine (WDT) in $\mathrm{NaCl}(10$ $\mu \mathrm{l} / \mathrm{g}$ body weight, i.p.) and perfused transcardially with $4 \%$ paraformaldehyde dissolved in $0.1 \mathrm{~m}$ phosphate buffer, $\mathrm{pH}$ 7.4. The survival time of 6-7 d after FG injection was chosen in line with previous data from adult rats (Hoover and Vertes, 2007), since similar investigations at neonatal age were not available. If positive staining was visible at P7-P8, we concluded that at the age of electrophysiological investigation the connectivity between the investigated areas was already present, even if we cannot definitively assess at which postnatal day within the time window of $\mathrm{P} 1-\mathrm{P} 5$ it emerged. When no positive staining was detected at P7-P8, we repeated the tracer injections at P5 to test whether the projections emerge shortly before the time point of electrophysiological investigation. The brains were removed and postfixed in the same solution for $24 \mathrm{~h}$. Blocks of tissue containing PFC, iHP, LEC, and VMT were sectioned in the coronal plane at $100 \mu \mathrm{m}$. The sections were air dried, coverslipped with Fluoromount-G (SouthernBiotech), and examined using an ultraviolet excitation filter of a Zeiss Imager M1 microscope. The photographs were adjusted for brightness and contrast using Adobe Photoshop CS6. To minimize the number of animals used (see the guidelines of the European Community Directive 2010/63/EU), part of the tracing data (see Fig. 5) was used both in the present study and in the study by Brockmann et al. (2011).

\section{Data analysis}

Detection and classification of neonatal patterns of oscillatory activity. The detection and classification of events has been achieved using a modified version of a previously developed unsupervised algorithm (Cichon et al., 2014) and has been confirmed by manual categorization.

Preprocessing of data. In a first step, the raw signals were processed by downsampling to $3255.2 \mathrm{~Hz}$ and bandpass filtering between 4 and $40 \mathrm{~Hz}$ using pop_eegfilternew of the EEGLAB toolbox (Swartz Center for Computational Neuroscience, La Jolla, CA) with automatic optimization of the filter order. The resulting activity was defined in all investigated brain regions as ground rhythm $[\mathrm{GR}(t)]$. Second, offsets and artifacts resulting from electrical interference or animal movement were automatically detected. Time windows with absolute values of a raw signal of $>980 \mu \mathrm{V}$ and its first derivative of $<15 \mathrm{nV} / \mu$ s were classified as offsets (i.e., the

\section{$\longleftarrow$}

(Figure legend continued.) The color-coded frequency plot shows the wavelet spectrum at an identical time scale. Inset, Segment of the theta burst displayed at a larger time scale (scale bar, $200 \mathrm{~ms}$ ). C, Average normalized power spectrum of oscillatory bursts in LEC ( $n=7$ animals). D, Timing relationships among neuronal firing and theta bursts in LEC. Di, Polar histogram (bin size, $1 / 20 \pi$ ) of the distribution of all spikes on the theta phase for all significantly phase-locked neurons $(n=11)$. The corresponding mean phase vector is displayed as a red line. Dii, Histogram of normalized theta amplitude envelope (red) and single-unit activity for two entorhinal neurons (SUA1, blue; SUA2, green) superimposed on the corresponding filtered (4-12 Hz) LFP (black). Note the confinement of increased neuronal firing to time windows of theta bursts. $\boldsymbol{E}$, Digital photomontage reconstructing the location of the Dil-labeled recording electrode (orange) in VMT of a Niss-stained $100-\mu \mathrm{m}$-thick coronal section from a P8 rat. The superimposed red dots mark the 16 recording sites, among which the deepest 4 are confined to RE. F, Characteristic theta burst from VMT displayed before (top) and after (4-30 Hz; middle) bandpass filtering, and the corresponding MUA after $300 \mathrm{~Hz}$ high-pass filtering (bottom). A color-coded frequency plot shows the wavelet spectrum on an identical time scale. $G$, Average normalized power spectrum of oscillatory bursts in VMT ( $n=7$ animals). $\boldsymbol{H}$, Timing relationship among neuronal firing and theta bursts in VMT. Hi, Polar histogram (bin size, $1 / 20 \pi$ ) of the distribution of all spikes from all neurons on the theta phase. The corresponding mean phase vector is displayed as a red line. Hii, Histogram of normalized theta amplitude envelope (red) and singleunit activity for two thalamic neurons (SUA1, blue; SUA2, green) superimposed on corresponding filtered (4-12 Hz) LFP (black). Note the confinement of increased neuronal firing to time windows of theta bursts. Inset, Plots of spike cluster isolation for SUA1 (blue, left) and SUA2 (green, right). Scale bars: $\boldsymbol{A}, \boldsymbol{E}, 1 \mathrm{~mm}$. In $\boldsymbol{C}$ and $\boldsymbol{G}$, the transparent area corresponds to SEM. signal reaches the maximum amplitude range) and were removed from the data. Fast electrical interference artifacts, which had a steep slope, were detected as having a maximum root mean square (RMS) of $>120$ $\mu \mathrm{V}$ and a large amplitude when compared with the ground rhythm. The slope threshold for artifact detection was set inversely proportional to the amplitude of the signal. Since artifacts resulting from animal movement have a high synchrony at all recording sites independently of brain region, we discarded all events with global cross-correlation values of $>0.8$.

Noise standard deviation and signal-to-noise ratio calculation. The moving SD of the noise over 1-s-long time windows was calculated according to the following formula:

$\left.\sigma_{\mathrm{GR}}(t)\right|_{\text {time_window }=1 \mathrm{~s}}$

$$
=\sqrt{\int_{-\infty}^{+\infty}\left(\mathrm{GR}(\tau)-\left.\overline{\mathrm{GR}(\tau)}\right|_{\text {time_window }=1 \mathrm{~s}}\right)^{2} \prod_{1 \mathrm{~s}}(t-\tau) d \tau}
$$

where

$$
\left.\overline{\operatorname{GR}(t)}\right|_{\text {time_window }=1 \mathrm{~s}}=\int_{-\infty}^{+\infty} \operatorname{GR}(\tau) \prod_{1 \mathrm{~s}}(t-\tau) d \tau,
$$

represents the moving average of $\mathrm{GR}(t)$ over the same time window. The SD of the noise was calculated by averaging the 10 lowest local minima of the $\left.\sigma_{\mathrm{GR}}(t)\right|_{\text {time_window }=1 \mathrm{~s}}$. The resulting values were used for the calculation of the signal-to-noise ratio (SNR) individually for each recording channel as follows:

$$
\mathrm{SNR}=20 \log \left(\frac{\operatorname{rms}(\mathrm{GR}(t))}{\sigma_{n}}\right) .
$$

The values of SNR varied between 8 and $18 \mathrm{~dB}$ for the recording channels in PFC, iHP, and LEC, as well as between 5 and $11 \mathrm{~dB}$ for VMT.

Detection and classification of oscillatory events. As previously reported (Brockmann et al., 2011), the threshold for detection of oscillatory events is proportional to the SD of the noise. To consider different SNRs at different recording sites, the detection threshold was corrected as follows:

$$
\text { threshold_correction_factor } r_{i}=\frac{\mathrm{SNR}_{\mathrm{i}}}{\max \left(\mathrm{SNR}_{\mathrm{i}}\right)} \text {. }
$$

Consequently, we defined the empirical formula for assessing the regionspecific threshold

threshold $_{\mathrm{i}}=\left(k \cdot \sigma_{n}\right) \cdot\left(0.75+0.25 \cdot\right.$ threshold_correction_factor $\left._{\mathrm{i}}\right)$,

where $k=4.2$.

Discontinuous oscillatory events were detected as deflections for which the RMS of the GR $(t)$ exceeds the calculated threshold for the region of interest. Only discontinuous slow events lasting at least $100 \mathrm{~ms}$ but not longer than $15 \mathrm{~s}$, containing at least three cycles, and having a peak-to-peak amplitude of $>1.4 \cdot$ threshold $_{i}$ were considered for analysis. Consecutive events with an interval of $>300 \mathrm{~ms}$ were considered as separate events.

For the classification of the detected oscillatory events, the $\mathrm{GR}(t)$ value was approximated by smoothing the signal with its moving average evaluated over a 10 -ms-long time window as follows:

$$
\widetilde{\mathrm{GR}(t)}=\left.\overline{\mathrm{GR}(t)}\right|_{\text {time_window }=10 \mathrm{~ms}}=\int_{-\infty}^{+\infty} \mathrm{GR}(\tau) \prod_{10 \mathrm{~ms}}
$$

$$
\times(t-\tau) d \tau \cong \mathrm{GR}(t) .
$$

All peaks and troughs of $\overline{\mathrm{GR}(t)}$ were detected to calculate the number of oscillatory cycles. As previously reported (Cichon et al., 2014), we discriminated between the prelimbic spindle burst (SB) and nested gamma 
spindle bursts (NG) according to their distinct features. The automatic classification of prelimbic activity patterns matched the manual categorization.

The discontinuous theta bursts in LEC and VMT were analyzed by their occurrence (defined as the number of bursts per minute), duration, maximum amplitude (defined as the voltage difference between the maximal positive and negative peaks), RMS amplitude, number of GR $(t)$ cycles, dominant frequency, as well as power in the theta $(4-12 \mathrm{~Hz})$ frequency band. The power density spectra were estimated using Welch's method with a $1 \mathrm{~s}$ Hanning window and a 0.5 overlapping factor. To remove the contribution of the $1 / f$ noise-to-power spectra, the power spectral density computed for oscillatory bursts was normalized by dividing it by the power spectral density computed for the noise (i.e., oscillation-free time windows). The dominant frequency was given by the peak in the power spectrum if its full-width at half-maximum was lower than $1 / 10$ of the considered bandwidth. Time-frequency plots were calculated by transforming the LFP events using a Morlet continuous wavelet. Minimal and maximal intensities in power were normalized to values between 0 and 0.5 , and were displayed in dark blue and red, respectively.

Assessment of correlation between discontinuous oscillatory patterns at different recording sites. The analysis was performed only for pairs of events with an onset difference $<0.5 \mathrm{~s}$. Two complementary methods were used. In the first instance, the maximal coherence as a spectral measure of correlation between two signals across frequencies was calculated from the cross-spectral density between the two signals and was normalized by the power spectral density of each (Jerbi et al., 2007). The computation was performed according to the following formula:

$$
C(f)=\frac{\left|\sum_{i=1}^{N} X_{\mathrm{i}}(f) Y_{i}^{*}(f)\right|^{2}}{\sum_{i=1}^{N}\left|X_{\mathrm{i}}(f)\right|^{2} \sum_{i=1}^{N}\left|Y_{\mathrm{i}}(f)\right|^{2}},
$$

where $X_{i}(f)$ and $Y_{i}(f)$ are the Fourier transforms of the signals $x$ and $y$ for the $i$ data segment at frequency $f$, and $*$ indicates the complex conjugate. The computations were performed using the magnitude-squared coherence function (MATLAB) based on Welch's averaged periodogram method $(0$ overlap, $1 \mathrm{~s}$ time window, $1 \mathrm{~Hz}$ frequency resolution). Coherence spectra were computed for filtered $(4-80 \mathrm{~Hz})$ data from all pairs of regions within the prelimbic-hippocampal-entorhinal and prelimbic-hippocampalthalamic networks. Frequency domains with significant coherence were determined by Monte Carlo simulation. For this, LFP segments of $1 \mathrm{~s}$ were shuffled 10,000 times, and the 95th percentile of the resulting distribution was used as a significance threshold. The maximal coherence coefficients in the theta frequency range $(4-12 \mathrm{~Hz})$ were quantified.

Second, a sliding window procedure was developed to calculate the cross-correlation coefficients for simultaneously recorded oscillations in all pairs of brain regions within the prelimbic-hippocampal-entorhinal and prelimbic-hippocampal-thalamic networks at specific time points across the entire data segment. Windows of $0.5 \mathrm{~s}$ in duration were moved across the data segment in increments of $0.2 \mathrm{~s}$, and their spectral analysis was performed for original and shuffled (oscillations recorded at different time points) data. Cross-correlation values ranging from -1 (anticorrelation) over 0 (no correlation) to 1 (maximal correlation) were color coded as blue and red, respectively.

Cross-correlation of instantaneous LFP amplitudes. To assess the causal interactions between pairs of brain areas, the squared cross-correlation of instantaneous amplitudes of network oscillations was calculated using a modified version of a model- and spike-independent method (Adhikari et al., 2010). For this, the LFPs in both regions were bandpass $(4-12 \mathrm{~Hz})$ filtered, and the instantaneous amplitudes of the filtered signal were calculated for time windows with co-occurring oscillations. The squared cross-correlation between these amplitudes was calculated, and the auto-correlation (including volume conduction effects) corresponding to zero lag peak was removed by applying a prewhitening filter. Peak lags were described as negative or positive using the hippocampal signal as a reference.
Table 1. Properties of theta bursts in LEC and VMT of neonatal rats

\begin{tabular}{lllll}
\hline Brain region & $\begin{array}{l}\text { Occurrence } \\
\text { (bursts/min) }\end{array}$ & $\begin{array}{l}\text { Amplitude } \\
(\mu \mathrm{V})\end{array}$ & Duration $(\mathrm{s})$ & $\begin{array}{l}\text { Main frequency } \\
(\mathrm{Hz})\end{array}$ \\
\hline LEC & $5.53 \pm 0.98$ & $231.16 \pm 9.88$ & $3.88 \pm 0.31$ & $7.4 \pm 0.4$ \\
VMT & $2.82 \pm 0.61$ & $85.39 \pm 4.27$ & $1.75 \pm 0.08$ & $6.05 \pm 0.6$ \\
\hline
\end{tabular}

Data are reported as the mean \pm SEM. Values were averaged for all recording sites confined to the investigated region ( $n=60-70$ events from $6-7$ rats).

Spike sorting and analysis. The raw signal was first high-pass filtered $(>407 \mathrm{~Hz})$. The threshold for the detection of multiple unit activity was individually set depending on the geometry of the recording site. As detailed previously (Nicolelis et al., 2003), the stored signals were sorted into similar waveform shapes using the Offline Sorter software (Plexon). For depicting the valid waveforms in $2 \mathrm{D} / 3 \mathrm{D}$ space, a combination of features (including the first three principal components, peak-to-peak voltage amplitudes) was chosen. Shapes of detected waveforms were visually inspected to exclude background noise. A group of similar waveforms was considered as being generated from a single neuron if it defined a discrete cluster in a 2D/3D space and exhibited a refractory period $(>1 \mathrm{~ms})$ in the interspike interval histograms. The assignment of spikes being generated by excitatory or inhibitory neurons, as performed in adults, is not feasible in neonatal/prejuvenile pups due to the absence of reliable differences in spike shapes during development. The quality of separation between identified clusters was assessed by the following four different statistical measurements: the classic parametric $F$ statistic of multivariate ANOVA (MANOVA), the J3 and PseudoF (PsF) statistics, and the Davies-Bouldin validity index (DB). The values of statistical testing ranged between $1.59 \times 10^{-17}$ and 0.65 for MANOVA, 0.012 and 10.14 for J3, 3.44 and 19674.49 for PsF, and 0.12 and 3.34 for DB.

Assessment of relationship between network oscillations and neuronal firing. The phase locking of spikes with the LFP was assessed using a previously described algorithm based on the Rayleigh $Z$ statistic (Siapas et al., 2005). If the firing of a neuron is modulated by the theta rhythm of the burst, then its phase over the theta cycle is not uniformly distributed. The significance of the unimodal preference was tested by Rayleigh $Z$ statistics. Phases of theta bursts were determined using the Hilbert transform (phase of 0 refers to the peak, and phase of $\pi$ refers to the trough of the cycle), and a specific phase was assigned to each spike based on the time of the occurrence of the spike. The spike trains were converted into a sequence of unit length vectors oriented by the phase values of their corresponding spikes. The orientation of the mean resulting vector was defined as the preferred phase. The phase locking between spikes and LFP was tested for significance using the Rayleigh statistic that is defined as $Z=n R^{2}$, where $R$ denotes the mean resultant length of the given phase series. The probability that the null hypothesis of sample uniformity holds is given by the following:

$$
P=e^{-z}\left[1+\left(2 Z-Z^{2}\right) /(4 n)-\left(24 Z-132 Z^{2}+76 Z^{3}-9 Z^{4}\right) /\left(288 n^{2}\right)\right] .
$$

For $n>50$, the approximation $P=e^{-Z}$ is adequate.

Assessment of unspecific synchrony. To decide on the presence of conduction synchrony between PL and VMT, the coherence between prelimbic spikes and thalamic LFP was calculated as previously described (Soteropoulos and Baker, 2006). Briefly, spike trains were converted to a waveform with the same sampling rate as the LFP by counting spikes in 2 ms sliding time windows for every sampling point. Coherence between the resulting waveform of the histogram and LFP was calculated. Only time windows corresponding to intermittent oscillations were used for further coherence analysis. The significance threshold was calculated for original versus shuffled (randomly distributed spikes before conversion to waveform) data.

Statistics. Data in the text are presented as the mean \pm SEM, and are displayed as bar diagrams, histograms, and polar plots. Statistical analyses were performed with SPSS version 15.0 (IBM)/SYSTAT (Systat Software). All values were tested for normal distribution by the Kolmogorov-Smirnov test, except their low number $(n<10)$ precluded reliable testing. For normally distributed values, either one-way ANOVA followed by Tukey post hoc analysis or paired/unpaired $t$ test was used. For a low number of values or not normally distributed values, nonparametric tests (Mann-Whitney- 
A
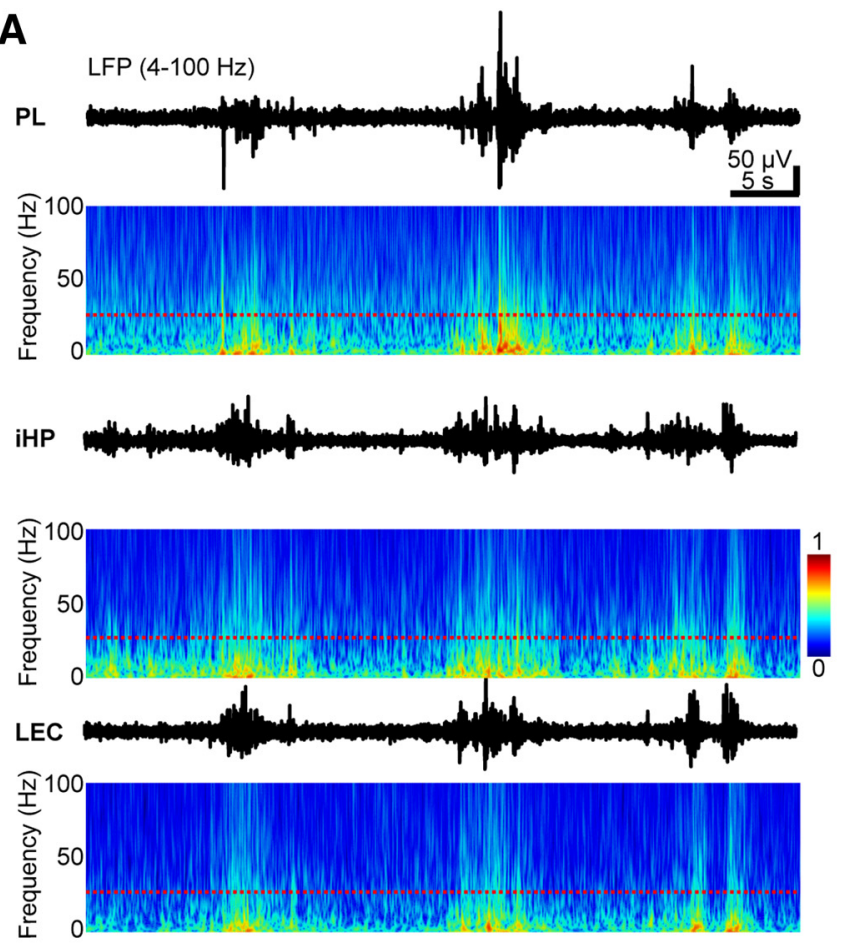

C

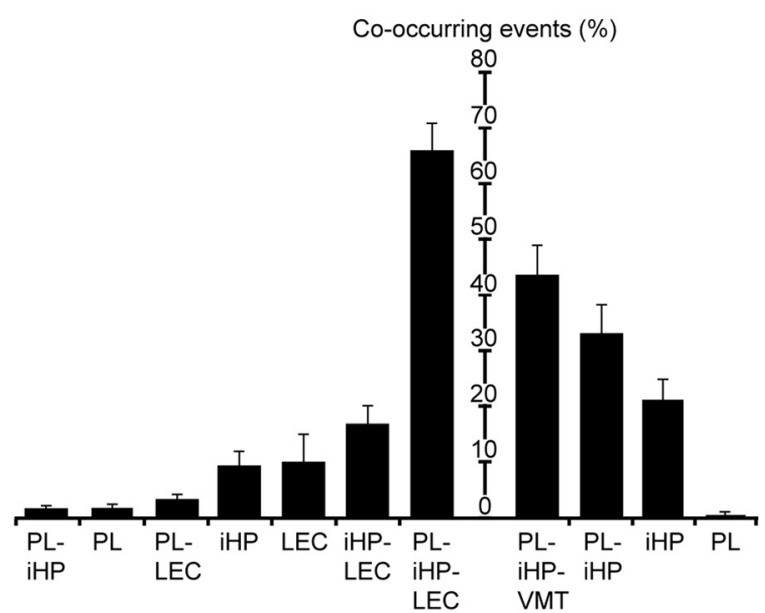

B

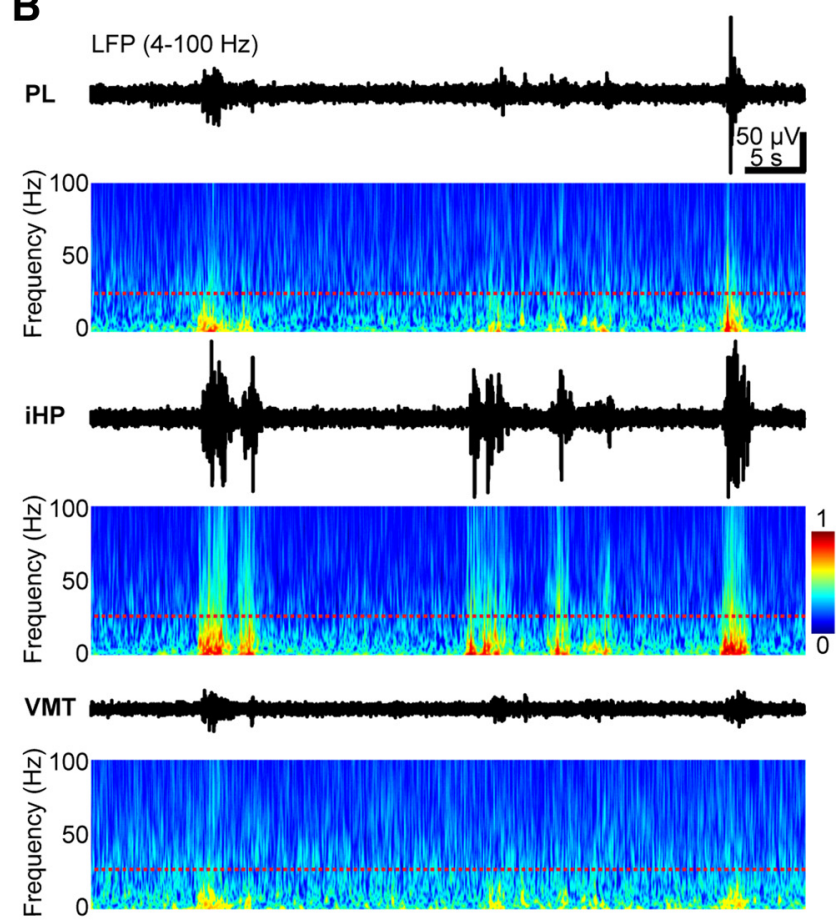

D (i)

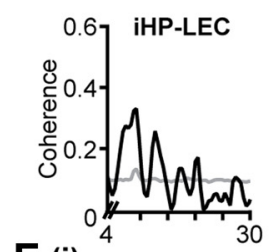

E (i)

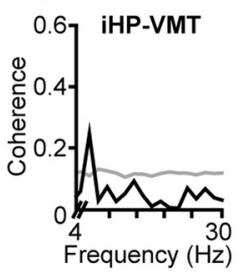

4

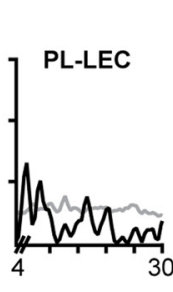

(ii)

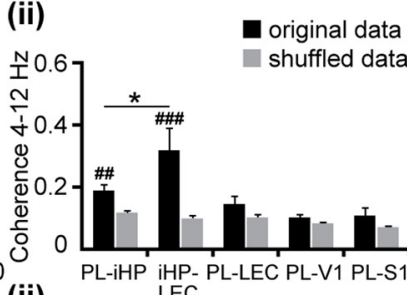

(ii)
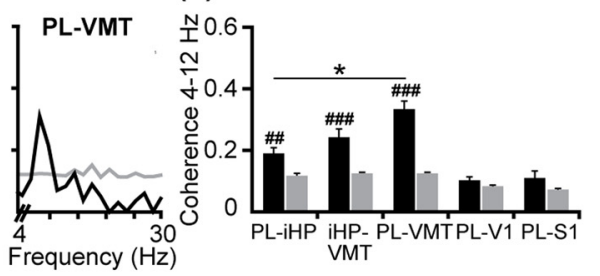

Figure 2. Oscillatory coupling by synchrony of neonatal PL, iHP, LEC, and VMT. $A$, Simultaneous LFP recordings of the discontinuous oscillatory activity in PL, hippocampal CA1 area, and LEC (layer II-III) accompanied by the color-coded wavelet spectra of the LFP at an identical time scale. Red line indicates the lower border of the gamma frequency range $(30 \mathrm{~Hz})$. Note the high incidence of simultaneously occurring events in all three brain regions. $\boldsymbol{B}$, Same as $\boldsymbol{A}$ for simultaneous LFP recordings of the oscillatory activity in PL, hippocampal CA1 area, and VMT nuclei. C, Bar diagram (mean \pm SEM) displaying the relative distribution of co-occurring events in PL, iHP, and LEC (left), as well as in PL, iHP, and VMT (right) normalized to the maximum number of events. D, Synchrony within prelimbic-hippocampal-entorhinal networks. Di, Coherence spectra (4-30 Hz) for simultaneously occurring oscillations (black) in iHP and LEC (left) or PL and LEC (right), as well as for shuffled data (gray) from a P7 rat. Note the significantly higher coherence values in the theta-alpha frequency range $(4-12 \mathrm{~Hz}$ ) for original data (black) when compared with shuffled data (gray). Dii, Bar diagrams (mean \pm SEM) displaying the max $4-12 \mathrm{~Hz}$ coherence for original data (black) and shuffled data (gray) recorded pairwise from prelimbic-hippocampal- entorhinal networks (for each pair, $n=60$ events from six pups) as well as for PL-V1 ( $n=120$ events from four pups) and PL-S1 ( $n=180$ from six pups). $E$, Same as $\boldsymbol{D}$ for synchrony within prelimbic-hippocampalthalamic networks ( $n=70$ events from seven pups) as well as PL-V1 and PL-S1. Note that for all areas the coherence for original data was higher when compared with shuffled data (Table 2). Significant differences within prelimbic- hippocampal- entorhinal or prelimbic-hippocampal-thalamic networks are marked by ${ }^{*}(p<0.05)$, whereas significant differences between coherence within prelimbic-hippocampal-entorhinal vs PL-S1 or within prelimbic-hippocampal-thalamic networks vs PL-S1 are marked by \#\# $(p<0.01)$ and \#\# $(p<0.001)$.

Wilcoxon test) were used. Significance levels of $p<0.05\left(^{*}\right), p<0.01\left({ }^{*}\right)$, or $\left.p<0.001{ }^{* * *}\right)$ were detected.

\section{Results}

Discontinuous patterns of coordinated oscillatory activity organize LEC and the nuclei of VMT in neonatal rats

We examined the activity patterns in LEC as well as in the nuclei of VMT by performing extracellular recordings of LFP and MUA in vivo from neonatal (P7-P9) rats $(n=23)$ under sleep-like conditions mimicked by light urethane anesthesia (Clement et al., 2008; Fig. 1). Our previous investigations showed that network activity and neuronal firing in cortical areas did not differ in urethane-anesthetized and nonanesthetized asleep rats (Yang et al., 2009; Bitzenhofer et al., 2015). Similar to HP, LEC develops during the first postnatal week by showing discontinuous patterns of network activity with a main frequency in the theta band ( $7.4 \pm 0.4 \mathrm{~Hz}$; normalized power within $4-12 \mathrm{~Hz}, 35.5 \pm 10.5$; vs normalized power within $12-30 \mathrm{~Hz}$ power, $12.6 \pm 3.3 ; n=7$ pups, $p<0.05$; Fig. 1C). Accordingly, these intermittent spindle- 
Table 2. Coherence for original vs shuffled data within networks PL-iHP-LEC and PL-iHP-VMT, and compared with coherence between PL-V1 and PL-S1

\begin{tabular}{|c|c|c|c|c|c|c|c|}
\hline Coherence & PL-iHP & iHP-LEC & PL-LEC & iHP-VMT & PL-VMT & PL-V1 & PL-S1 \\
\hline Original & $0.19 \pm 0.02$ & $0.32 \pm 0.07$ & $0.14 \pm 0.03$ & $0.24 \pm 0.03$ & $0.33 \pm 0.03$ & $0.1 \pm 0.01$ & $0.11 \pm 0.02$ \\
\hline Shuffled data (significance threshold) & $0.12 \pm 0.01$ & $0.1 \pm 0.09$ & $0.1 \pm 0.01$ & $0.12 \pm 0.004$ & $0.12 \pm 0.003$ & $0.08 \pm 0.005$ & $0.07 \pm 0.003$ \\
\hline$p$ values (vs PL-V1, PL-S1, respectively) & $0.026,0.003$ & $0.006,<0.0001$ & $0.942,0.622$ & $0.038,0.034$ & $0.036,0.032$ & & \\
\hline
\end{tabular}

Values are reported as the mean \pm SEM, unless otherwise indicated, for $n=6$ pups (network PL-iHP-LEC), $n=7$ pups (network PL-iHP-VMT), $n=4$ pups (PL-V1), and $n=6$ pups (PL-S1).

A

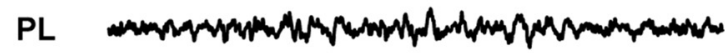

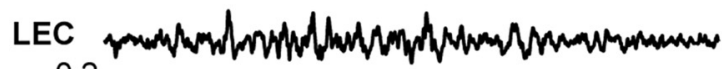

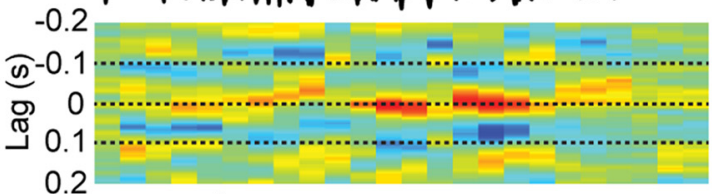

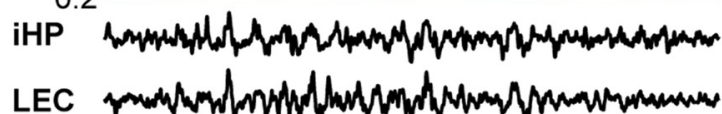

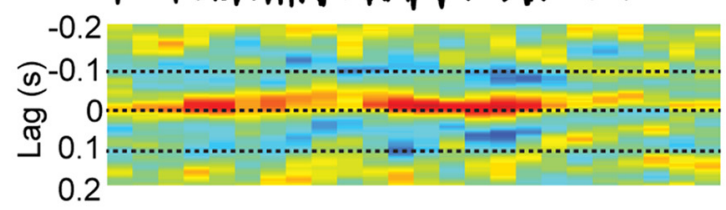

B
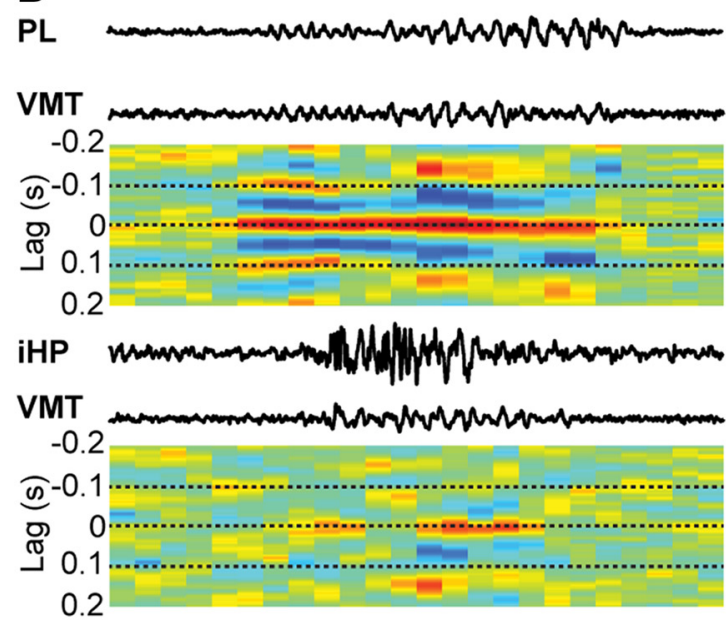

Shuffled data
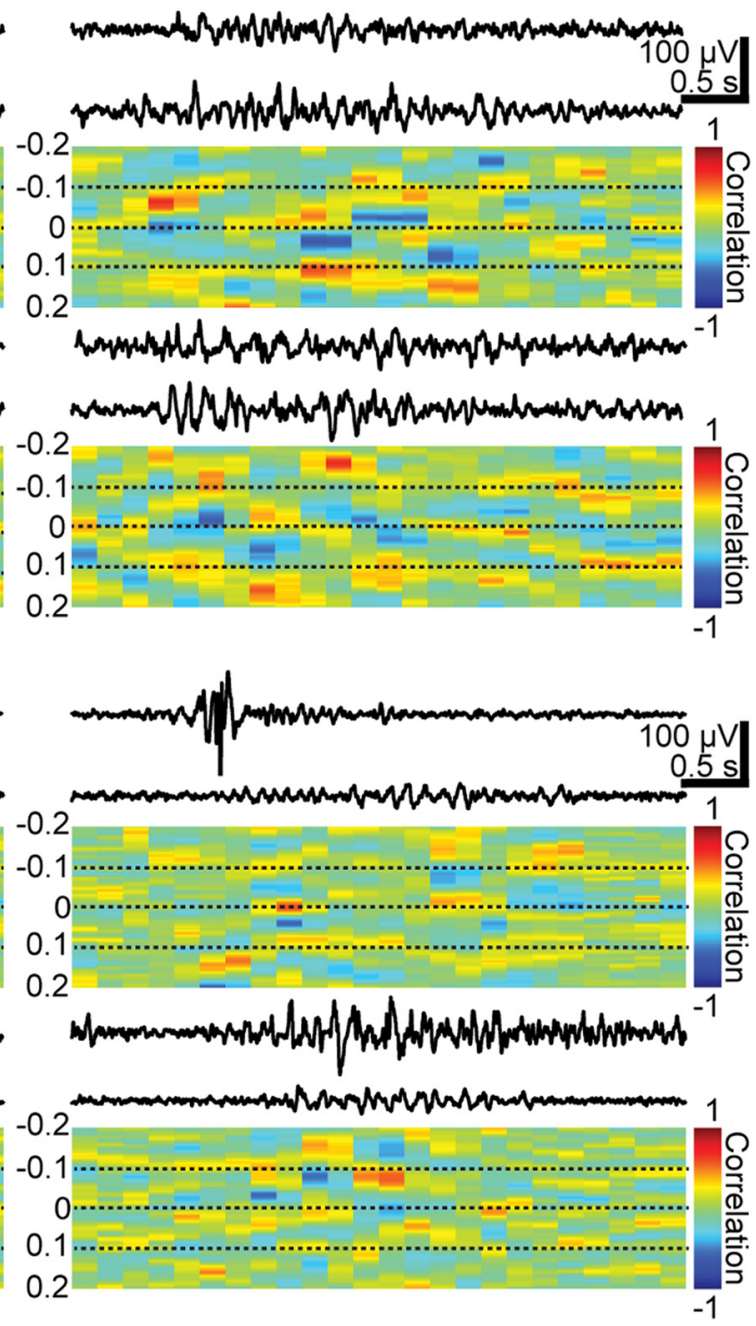

Figure 3. Dynamics of oscillatory coupling during discontinuous oscillations in neonatal PL, iHP, LEC, and VMT. A, Simultaneous LFP recordings (4-80 Hz) of oscillatory activity in PL and LEC (top) as well as iHP and LEC (bottom) of a P8 rat accompanied by the time-aligned sliding window cross-correlograms for original (left) and shuffled (right) data. Note the high and sustained correlation between iHP and LEC. B, Same as A for oscillatory activity in the PL and VMT as well as in the iHP and VMT. Note the sustained coupling within prelimbic-thalamic networks. All colors other than green represent a significant positive (red) or negative (blue) correlation.

shaped field oscillations were defined as theta bursts and resembled the activity previously reported in MEC for neonatal mice (Unichenko et al., 2015). When recorded from multiple recording sites covering layers II/III, the entorhinal theta bursts had a relative short duration and small amplitude (Table 1$)$. They were accompanied by single-unit activity (SUA) discharge $(0.75 \pm$ $0.17 \mathrm{~Hz}, n=11$ cells from four pups). The firing rates of individual neurons were highest during theta bursts $(3.2 \pm 0.54 \mathrm{~Hz})$, whereas firing rates during oscillation-free time windows were very low (Fig. 1Dii). Examination of the relationship between the SUA and phase of theta bursts showed that all investigated entorhinal neurons ( $n=11$ cells from four pups) fired shortly before the peak of theta cycle (Fig. 1Di).
Toward the end of the first postnatal week, the VMT nuclei also expressed coordinated network oscillations (Fig. 1E,F). Their spectral distribution with a dominant frequency in the theta band $(6.05 \pm 0.6 \mathrm{~Hz}$; normalized power within $4-12 \mathrm{~Hz}$ : $15.9 \pm 7.6$ vs normalized power within $12-30 \mathrm{~Hz}: 6.1 \pm 2.7 ; n=$ 7 pups; Fig. $1 G$ ), which was similar to that of hippocampal and entorhinal oscillations, led to their classification as theta bursts. However, the thalamic theta bursts occurred less frequently $(2.82 \pm 0.61$ events $/ \mathrm{min})$ and at a significantly $(p<0.001)$ lower amplitude $(85.39 \pm 4.27 \mu \mathrm{V}, n=76$ events from seven pups) when compared with entorhinal events (Table 1). Even if the number and spiking rates of thalamic neurons were very low, reflecting their immaturity, the firing peaked during but were not 


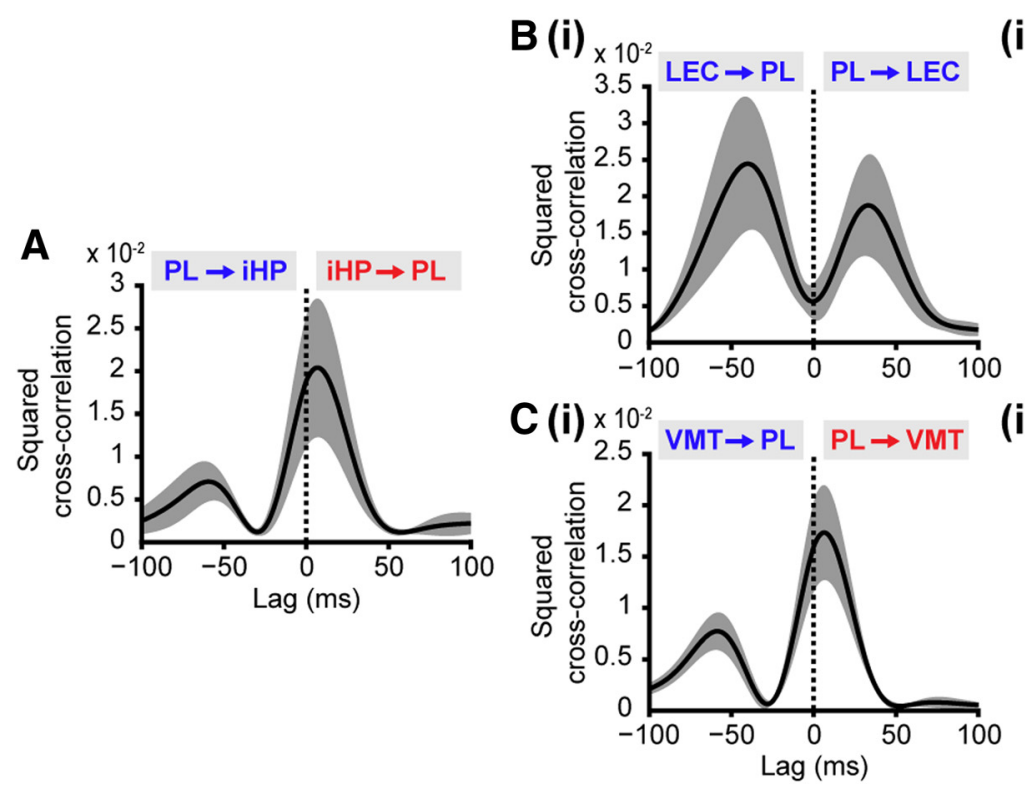

(ii)

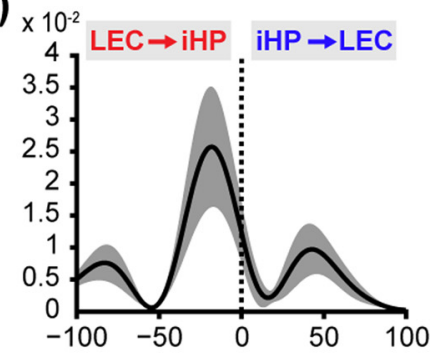

(ii) $\times 10^{-3}$

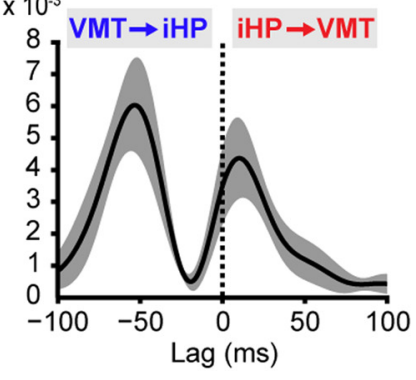

Figure 4. Directed interactions among neonatal PL, iHP, LEC, and VMT. A, Squared cross-correlations of the amplitudes of theta-filtered LFP recorded from PL and iHP and averaged for all investigated rats $(n=14)$. Positive time lags of the peaks correspond to iHP leading PL. B, Same for LEC and PL $(n=8 ; \boldsymbol{B i})$ as well as LEC and iHP ( $n=8 ; \boldsymbol{B i i})$. C, Same for VMT and PL $(n=6$; $\boldsymbol{C} i)$ as well as VMT and $\mathrm{iHP}(n=6$; (ii). Short $(<20 \mathrm{~ms})$ lags of significant peaks correspond to putative monosynaptic interactions and are marked in red, whereas long ( $20-70 \mathrm{~ms})$ lags correspond to putative polysynaptic interactions and are marked in blue. Data are shown as the mean \pm SEM.

phased locked with theta bursts in VMT $(n=2$ cells from seven pups; Fig. $1 F, H)$.

Thus, discontinuous theta oscillations with distinct properties and relationships to firing activity represent the dominant pattern of activity in neonatal LEC and VMT.

\section{Oscillatory activity provides tight coupling of LEC and VMT within neonatal prelimbic-hippocampal networks}

To assess the role of early entorhinal and thalamic activity for the functional communication within neonatal prefrontalhippocampal networks (Brockmann et al., 2011), we investigated the interactions among PFC, iHP, and LEC or among PFC, iHP, and VMT by using several approaches. The entire investigation focused on PL, which is the prefrontal subdivision that receives the strongest hippocampal drive. In a first step, we examined the temporal correspondence of discontinuous oscillations recorded simultaneously from all three areas (Fig. 2). We previously characterized the theta bursts recorded from stratum pyramidale of the hippocampal CA1 area (i.e., discontinuous oscillations with a main frequency of $\sim 7 \mathrm{~Hz}$ ) as well as the prelimbic SBs (i.e., discontinuous 7-10 Hz spindle-shaped oscillations) and NGs (i.e., spindle-shaped oscillations with frequency alternating between theta and beta-to-low gamma frequency that are superimposed with high-frequency oscillations) recorded from layer II/ III of the PL (Brockmann et al., 2011). The majority of hippocampal and entorhinal theta bursts occurred within a narrow time window $(<3 \mathrm{~s})$ with SBs or NGs in PL $(65.67 \pm 5.2 \%$; $n=328$ events from six pups; Fig. $2 A, C$ ) and were defined as co-occurring events. Few oscillations were restricted to only two areas, the highest incidence being observed within hippocampalentorhinal networks $(16.5 \pm 3.6 \%, n=328$ events from six pups). Entorhinal oscillatory events with no temporal correspondence either in iHP or PL were detected, but represented only $\sim 10 \%$ of the total number of bursts. Similarly, a large fraction of discontinuous oscillatory events $(43.64 \pm 5.4 \%$; $n=232$ events from seven pups) co-occurred in PL, iHP, and nuclei of VMT (Fig. 2B,C). Thalamic theta bursts with no prelimbic and/or hippocampal correspondent were never observed (Fig. 2C).

In a second step, we assessed the temporal synchrony between co-occurring oscillatory events by performing spectral coherence analysis (Fig. 2D,E). Solely for theta-alpha frequencies $(4-12$ $\mathrm{Hz}$ ), the coherence of co-occurring oscillations was higher than the significance threshold (shuffled data; Fig. 2Di,Ei; Table 2). Focusing on the synchrony within prelimbic-hippocampalentorhinal networks, the highest coherence was obtained between iHP and LEC $(0.32 \pm 0.07, n=6$ pups $)$, whereas the synchrony between LEC and PL ( $0.14 \pm 0.03, n=6$ pups) was comparable to the previously reported coupling within prelimbic-hippocampal networks $(0.19 \pm 0.02, n=13$ pups; Fig. 2 Dii). In contrast, when focusing on the synchrony among PL, iHP, and VMT nuclei, the tightest coupling was detected between PL and VMT $(0.33 \pm 0.03, n=7$ pups; Fig. 2 Eii). In line with previous investigations (Brockmann et al., 2011), this high level of coherence is a genuine feature of investigated neonatal networks, because (1) the synchrony between PL and other brain areas, such as V1 or S1, was significantly lower than the coherence values calculated for all pairs of regions, except the PL-LEC (Table 2); and (2) the "true synchrony" (Lachaux et al., 1999), which was measured as coherence between spikes in one area (e.g., PL) and LFP in the other area (e.g., VMT), was significantly higher when compared with time-shuffled data $(0.38 \pm 0.007$ vs $0.36 \pm 0.007, p=$ $0.03)$. These data suggest that the contribution of nonspecific or conduction synchrony, if any, is minor.

In a third step, we assessed the coupling dynamics throughout individual events by estimating the synchrony during discontinuous oscillations co-occurring in PL, iHP, and LEC as well as in PL, iHP, and VMT. Cross-correlation analysis of prelimbic SB/NG as well as hippocampal and entorhinal theta bursts revealed that the first peak of individual cross-correlation $(\sim 0 \mathrm{~s}$ lag $)$ fluctuated systematically and periodically for both PL-LEC and iHP-LEC oscillations as a sign of transient coupling and decoupling of these cortical regions (Fig. 3). Similarly, the strength of coupling between thalamic nuclei and either PL or iHP varied during oscillatory events. However, con- 


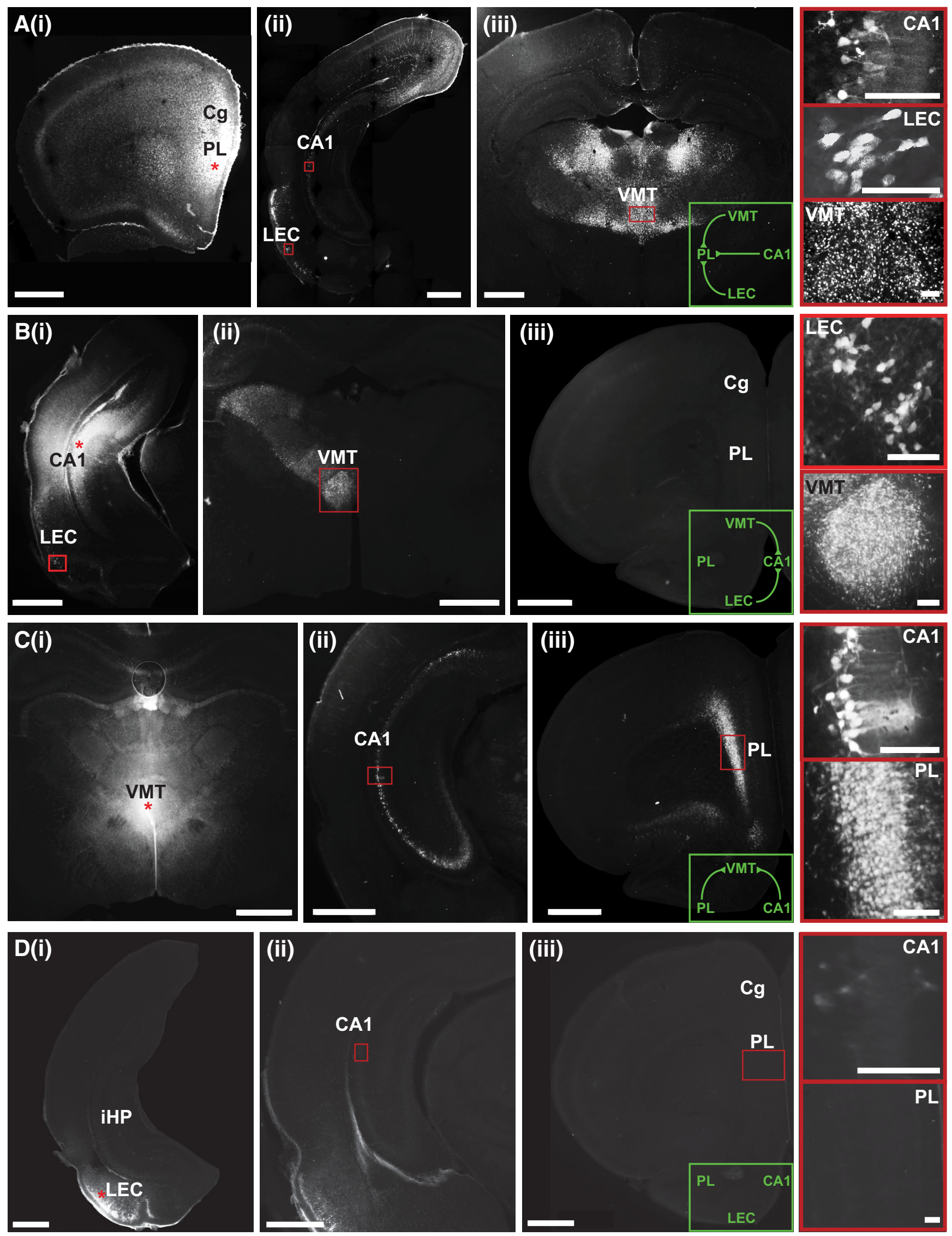

Figure 5. Long-range monosynaptic axonal projections connecting neonatal PFC, hippocampal CA1, LEC, and the nuclei of VMT. $A$, Photomicrographs depicting retrogradely labeled neurons in the hippocampal CA1 area and LEC (Aii) as well as in the VMT nuclei (Aiii) of a P7 rat after FG injection into PL (red star) at P1 (Ai). The tracing data used for Ai and Aii are from Brockmann et al., (2011). B, Photomicrographs depicting retrogradely labeled neurons in LEC (Bi) and VMT (Bii) of a P7 rat after FG injection into CA1 (red star) at P1 (Bi). Note the (Figure legend continues.) 
stantly high cross-correlation during the entire burst was calculated for $36.67 \%$ of hippocampal-entorhinal oscillations and for $51.67 \%$ of prelimbic-thalamic oscillations, but for only $6.67 \%$ of prelimbicentorhinal and $11.67 \%$ of hippocampal-thalamic bursts. Confirming the coherence analysis, these results indicate a tight coupling within hippocampal-entorhinal and prelimbic-thalamic networks.

Due to the symmetric interdependence of cross-correlation and coherence, they do not offer reliable insights into the direction of information flow between two brain areas. To assess the directed interactions among PL, iHP, LEC, and VMT, we calculated the squared cross-correlation of instantaneous amplitudes of filtered LFP for pairs of brain areas and determined the time lag between them. By these means, directed interactions are reliably estimated independently of spike sampling, amplitude variations, and noise-sensitive autoregressive modeling (Adhikari et al., 2010). The contribution of autocorrelation and unspecific volume conduction leading to false-positive peaks at a $0 \mathrm{~ms}$ time lag was subtracted. Confirming the monosynaptic drive from the CA1 area to PL previously identified by Granger causality analysis (Brockmann et al., 2011), the maximal squared cross-correlation between PL and $\operatorname{iHP}(0.019 \pm 009)$ was obtained at a positive time lag of $6.8 \pm 1.2 \mathrm{~ms}$ (Fig. $4 A$ ). Additionally, feedback coupling seems to indirectly relay information from PL back to iHP, because a small, but significant, peak of cross-correlation was detected at a negative time lag of $-59.6 \pm 3.2 \mathrm{~ms}$. Directed interactions equally entrained LEC with PL and iHP. While crosscorrelation peaks of comparable magnitude $(0.018 \pm 0.008$, $0.024 \pm 0.008)$ at rather long delays $(33.2 \pm 2.1 \mathrm{~ms},-40.1 \pm 3.4$ $\mathrm{ms}$ ) indicate that PL and LEC couple via reciprocal and, most likely, polysynaptic interactions (Fig. $4 B i$ ), the prominent peak $(0.025 \pm 0.01)$ at short delay $(-17.7 \pm 2.4 \mathrm{~ms})$ corresponding to LEC $\rightarrow$ iHP reflects a strong entorhinal drive on hippocampal theta bursts (Fig. 4Bii). Correspondingly, the firing of individual hippocampal neurons was precisely timed by the phase of entorhinal theta bursts. Cross-correlation analysis of LFP amplitudes revealed different coupling of VMT within prelimbic-hippocampal networks. Prelimbic activity drives the thalamic theta bursts via putative monosynaptic projections, because a prominent crosscorrelation peak $(0.017 \pm 0.006)$ was present at a short $(6.3 \pm 1.3$ $\mathrm{ms}$ ) positive time lag (Fig. 4Ci). On its turn, VMT seems to relay the prelimbic drive and strongly interact with $\mathrm{iHP}$, as shown by the peak of cross-correlation $(0.006 \pm 0.002)$ at negative time lag $(-53 \pm 3.7$ $\mathrm{ms}$ ) that corresponds to a drive VMT $\rightarrow$ iHP (Fig. 4Cii). These putative polysynaptic interactions were accompanied by a reciprocal putative monosynaptic drive iHP $\rightarrow$ VMT, with the squared crosscorrelation $(0.004 \pm 0.001)$ peaking at a short positive time lag $(9.8 \pm 1.6 \mathrm{~ms})$.

These data indicate that despite similar coupling by synchrony, LEC and VMT seem to have different contributions to directed communication within neonatal prelimbic-hippocampal networks.

\section{$\leftarrow$}

(Figure legend continued.) absence of retrogradely labeled cells in PL (Biii). C, Photomicrographs depicting retrogradely labeled neurons in the hippocampal CA1 area (Cii) of a P7 rat after FG injection into VMT (red star) at P1 ( $\boldsymbol{C}$ ) as well as labeled neurons in PL (Ciii) after FG injection into VMT at P5. Note the confinement of staining to the deeper layers of PL. D, Photomicrographs depicting the FG injection site (red star) in LEC at P1 (Di) and the absence of retrogradely stained neurons in the hippocampal CA1 area (Dii) and PL (Diii) at P7. For $\boldsymbol{A}-\boldsymbol{D}$, scale bars correspond to $1 \mathrm{~mm}$, and areas marked with red boxes are displayed at higher magnification in the right panel. Scale bars, $100 \mu \mathrm{m}$. For Aiii-Diii, the insets (green) depict the summary of axonal projections revealed after FG injection into the area shown in $\boldsymbol{i}$.

\section{Axonal projections are the anatomical substrate of directed interactions of LEC and thalamic nuclei within prelimbic- hippocampal networks}

While the time lags of cross-correlation peaks give the first insights into the substrate of directed interactions among PL, iHP, LEC, and VMT, they need confirmation by morphological assessment of axonal pathways among these areas. Whereas the connectivity patterns of LEC and VMT have been extensively investigated in adults (McKenna and Vertes, 2004; Vertes, 2006; Hoover and Vertes, 2007), their spatial and temporal profiles during development remain largely unknown. We previously showed that already at the end of the first postnatal week hippocampal axonal projections strongly innervate PL, whereas no direct connections from PL back to the hippocampal CA1 area could be detected (Brockmann et al., 2011). To elucidate the anatomical integration of LEC and VMT within the neonatal prelimbic-hippocampal networks, we injected small amounts of the retrograde tracer FG into each area of interest ( $n=17$ pups). First, FG injection centered over PL of P1 pups $(n=3)$ led, after $6 \mathrm{~d}$, to the labeling of cells in the CA1 area of the intermediate and ventral HP (Fig. 5A). Moreover, stained neurons, especially in layers II/III of LEC were detected (Fig. 5Aii). Of note, the entorhinal neurons projecting to PL appeared clustered into columnlike groups of neighboring cells (Fig. $5 \mathrm{Aii}$, inset), which have not been described in adult LEC (Hoover and Vertes, 2007) and the function of which remains to be elucidated. Dense retrograde staining after FG injection in PL was also obtained in VMT nuclei (Fig. 5Aiii). The thalamic neurons projecting to PL lacked a specific spatial organization.

In a second step, we confined the FG injection to the intermediate and ventral HP ( $n=4$ pups; Fig. $5 B)$. Detailed investigation of consecutive sections was performed to exclude the possibility that unspecific leakage of tracer causes staining of entorhinal neurons. While both LEC and VMT projected to iHP, the entire PFC lacks retrogradely stained neurons (Fig. 5Biii), confirming the absence of direct projections from PL to HP (Brockmann et al., 2011). In a third step, FG injection in VMT ( $n=5$ pups) at $P 5$, but not at P1 revealed prominent staining in the hippocampal CA1 area as well as in PL (Fig. 5C). However, the prelimbic neurons projecting to VMT were not homogeneously distributed, but exclusively confined to layers V and VI (Fig. 5Ciii), similarly to the connectivity patterns observed in adult rats (McKenna and Vertes, 2004). Finally, we assessed the projections targeting neonatal LEC ( $n=5$ pups). Injection of FG into LEC of P1 rats led to no retrograde staining in either iHP or PL. To test whether the hippocampal and prelimbic projections to the EC develop later during development and, therefore, are absent shortly after birth, we additionally performed FG injections at P5 (Fig. 5D). At the end of the first postnatal week, LEC still did not receive direct afferents from PL and iHP. Since these projections have been identified in adult rats as a substrate of functional network communication (Kerr et al., 2007), they are likely to emerge during the prejuvenile or juvenile development, a time window beyond the focus of the present investigation. These findings suggest that already at the end of the first postnatal week monosynaptic axonal projections link LEC and VMT with PL and iHP. They might represent the substrate of coupling by synchrony and directed interactions revealed by amplitude cross-correlation.

\section{Reversible silencing of PL or VMT selectively perturbs the neonatal network communication}

In line with the directed communication resolved by amplitude cross-correlation, LEC and VMT seem to have different roles for 
the activation of neonatal prelimbic-hippocampal networks. To confirm these data, we reversibly blocked the neuronal firing in PL ( $n=11$ pups), and investigated the consequences of manipulation on the activity patterns of iHP, LEC, and VMT in P7-P8 rats. For this, the action potential blocker lidocaine (300 nl, 200 $\mathrm{nl} / \mathrm{min}$ ) was injected into PFC. Confirming our previous data (Brockmann et al., 2011) and in line with the spherical volume calculation (Tehovnik and Sommer, 1997), lidocaine acted on a radius of 295-425 $\mu \mathrm{m}$ in PL. Here, it led to a significant reduction of the discontinuous burst activity by $67.82 \pm 8.72 \%$ and of the MUA frequency by $87.07 \pm 11.21 \%(n=11$ pups $)$, which lasted for $\sim 40 \mathrm{~min}$ (Fig. $6 A-C$ ). Moreover, the prelimbic bursts had a significantly lower amplitude (before lidocaine, $196.9 \pm 13.4 \mu \mathrm{V}$; lidocaine, $134.25 \pm 16.38 \mu \mathrm{V} ; p=0.01$ ), duration (before lidocaine, $1.88 \pm 0.09 \mathrm{~s}$; lidocaine, $1.36 \pm 0.16 \mathrm{~s} ; p=0.003)$, and oscillatory power between 4 and $12 \mathrm{~Hz}$ and between 12 and $30 \mathrm{~Hz}$ (before lidocaine, $4.95 \pm 0.78$ and $0.87 \pm 0.34 \mu \mathrm{V}^{2} / \mathrm{Hz}$; lidocaine, $1.95 \pm 0.25$ and $0.19 \pm 0.03 \mu \mathrm{V}^{2} / \mathrm{Hz} ; p=0.0020$ and $p=$ 0.0098 , respectively; Fig. $6 B, C)$. This transient silencing of $\mathrm{PL}$ had major effects on the thalamic activity, leading to a significant $(p=0.042)$ decrease in the occurrence of theta bursts by $62.93 \pm$ $16.89 \%$ (Fig. 6Bi). The amplitude, duration, and spectral distribution of network oscillations in VMT were not affected by lidocaine treatment (Fig. 6Biii). While on average ( $n=5$ rats) the properties of hippocampal theta bursts were not significantly changed after lidocaine application, we detected a clear correlation (Pearson's correlation, 0.85 ) between the magnitude of inactivation in PL and iHP for individual rats (Fig. 6Bii). In animals with an inactivation magnitude of $>70 \%$, the occurrence of hippocampal theta burst was also strongly reduced by $>34 \%$. In contrast, when lidocaine had a rather moderate effect in the PL (36-58\%), the hippocampal activity remained largely unaffected. Of note, the inactivation of PL had no significant effects on the entorhinal theta burst at the group ( $n=6$ rats) and individual level (Pearson's correlation for relative changes of occurrence, 0.12; Fig. 6Bii).

Thus, the prelimbic activity seems to directly contribute to the network entrainment in VMT and, to a weaker extent, to hippocampal theta bursts, but does not directly impact LEC. To confirm the role of VMT as a link between iHP and PL, we reversibly blocked the thalamic neuronal firing by injecting lidocaine into VMT, and simultaneously recorded the oscillatory activity in PL, iHP, and VMT (Fig. 6D-F). Lidocaine reduced the theta bursts in VMT by $48.92 \pm 7.52 \%$ ( $n=6$; Fig. $6 E)$. This transient silencing of VMT had major effects on the prelimbic and hippocampal activity, leading to a significant ( $p=0.028, p=0.046)$ decrease of the occurrence of oscillatory events by $41.24 \pm 7.82 \%$ and $25.78 \pm 7.62 \%$, respectively (Fig. 6E). The amplitude, duration, and spectral distribution of network oscillations within prelimbic-hippocampal-thalamic networks were not affected by lidocaine treatment of VMT. While the coherence between PL and iHP was not affected by the lidocaine silencing of VMT (control, $0.18 \pm 0.02$; after VMT inactivation, $0.15 \pm 0.04 ; p=0.60, n=5$ pups), a significant ( $p=0.025$ ) decrease of the peak of squared crosscorrelation corresponding to putative polysynaptic feedback interactions was detected (before lidocaine, $0.029 \pm 0.009$; after lidocaine, $0.006 \pm 0.001$; Fig. $6 F$ ). These data suggest that VMT functionally links PL and iHP, being activated by prelimbic network oscillations and further relaying this activation to iHP via putative polysynaptic interactions.

\section{Discussion}

The coactivation of prefrontal-hippocampal networks emerges during neonatal development with a theta drive from the intermediate-ventral hippocampal CA1 area to PL (Brockmann et al., 2011). In the absence of direct anatomical and functional feedback at this early age and taking into account that unidirectional communication in the brain occurs rather seldom, it is likely that the prefrontal control of hippocampal excitatory drive involves other brain areas as relay stations. At adulthood, both LEC and the nuclei of VMT have been identified as tightly interacting with PFC and HP during memory-related directed communication (Morrissey et al., 2012). Here, we used multielectrode recordings in vivo, connectivity tracing, and pharmacological silencing to elucidate the role of entorhinal and thalamic activation for the oscillatory coupling of prefrontal-hippocampal networks in neonatal rats. We demonstrate that (1) coordinated patterns of discontinuous oscillatory activity peaking in theta frequency band already start to entrain LEC and the nuclei of VMT during the first postnatal week; (2) similar to memory-related activation at adulthood, neonatal entorhinal activity mainly controls the hippocampal theta bursts by timing the neuronal firing via monosynaptic axonal projections; and (3) theta-gamma activity of neonatal PL directly controls the oscillatory entrainment of VMT, which, in turn, may drive the activation of intermediate hippocampal CA1 area via polysynaptic reciprocal projections. These data show that even if anatomical connections within prefrontal-hippocampal-entorhinal and prefrontalhippocampal-thalamic networks are in place at a neonatal age, they do not always serve as a substrate for functional interactions. The immaturity, different origin, or sparseness of axonal projections from VMT to HP as well as from LEC to PL may account for the involvement of additional brain areas, as revealed by the low coherence and long time lags in causality analysis. Together, the results suggest that LEC and VMT have different roles within neonatal prefrontal-hippocampal networks. While LEC strongly communicates with iHP and facilitates the emergence of its discontinuous theta bursts, the thalamic nuclei might act as a relay station, ensuring feedback communication from PL to iHP (Fig. 7).

\section{Coordinated patterns of network activity in the lateral entorhinal cortex and "nonspecific" thalamic nuclei at neonatal age versus adulthood}

Based on its afferents and efferents (Canto et al., 2008), the adult entorhinal cortex is considered to be the gatekeeper for hippocampal formation (Kerr et al., 2007). The type of information that is preprocessed before being directed to HP seems to differ for LEC and MEC. While MEC mainly encodes spatial information (Fyhn et al., 2004) by recurrent inhibitory circuitry (Couey et al., 2013; but see also Buetfering et al., 2014), LEC is mainly responsible for nonspatial information about content (Knierim et al., 2014), including emotional significance (Morrissey et al., 2012). These specific functions and the corresponding network interactions emerge relatively late during postnatal development (Bjerknes et al., 2015; Tan et al., 2015). For example, stable grid cell firing has an abrupt onset at P20 after eye opening and the start of explorative behavior (Wills et al., 2012). Already before eye opening, head direction cells are present in HP and connected areas (Tan et al., 2015). In vitro investigations suggested that even earlier MEC generates spontaneous bursting activity, which has been proposed to refine the neonatal circuitry (Sheroziya et al., 2009). The present in vivo data reveal that the early patterns of coordinated activity in LEC drive the theta entrainment of iHP 


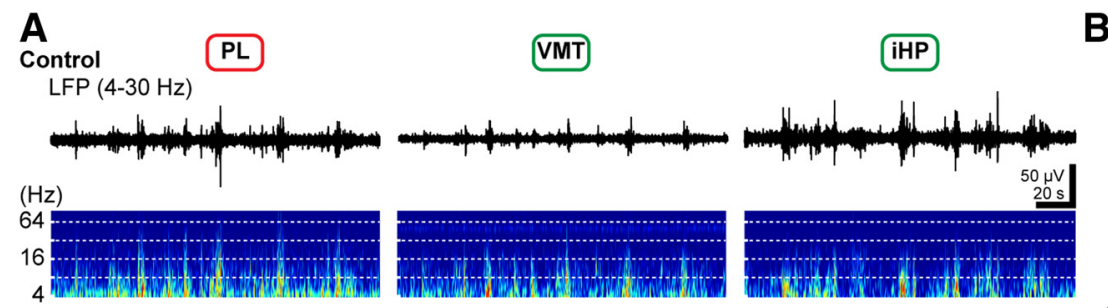

B (i)

(ii) Relative change PL (\%)
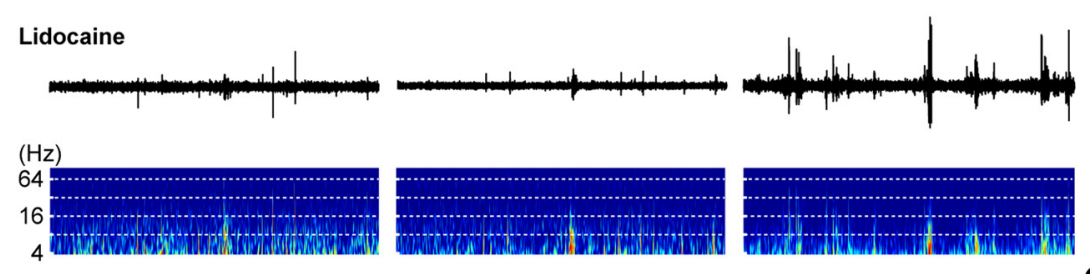

(iii)
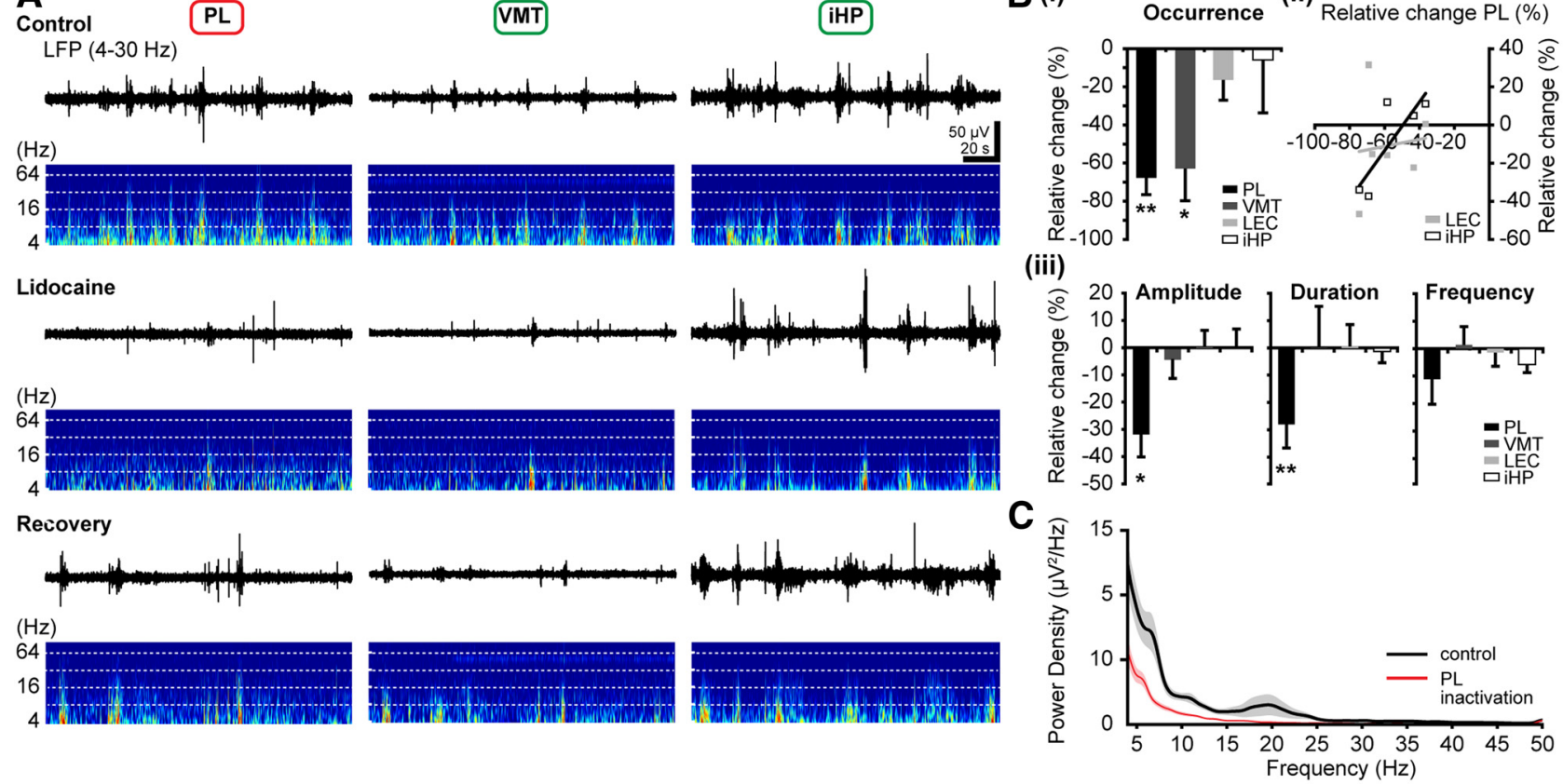

D Control

VMT $\operatorname{LFP}(4-30 \mathrm{~Hz})$

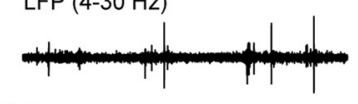

$(\mathrm{Hz})$

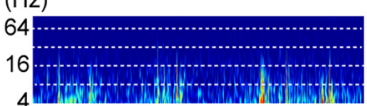

Lidocaine

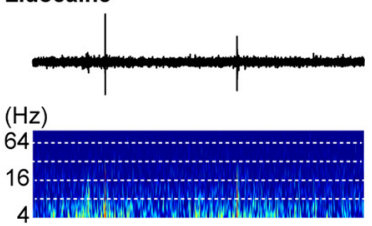

Recovery

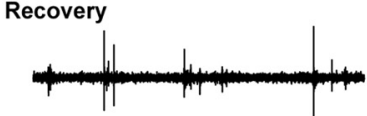

$(\mathrm{Hz})$

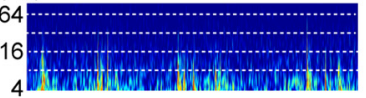

$\mathrm{PL}$
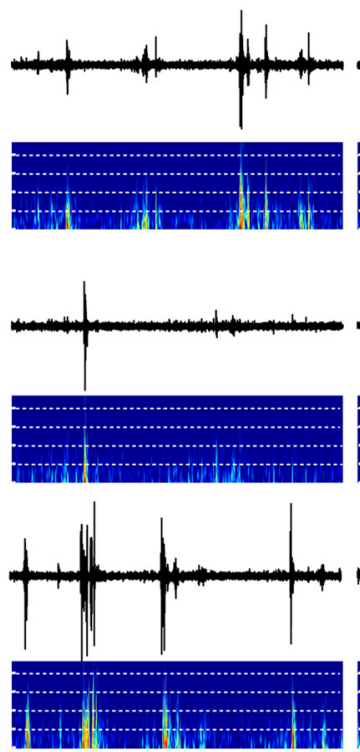

iHP
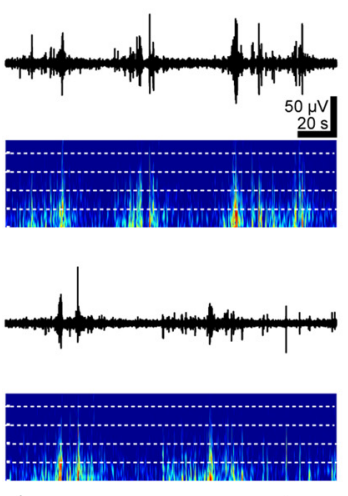

$E$ (i)

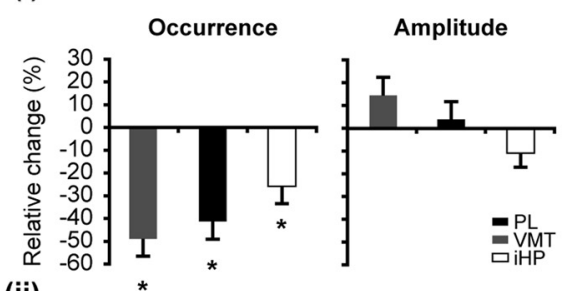

(ii)

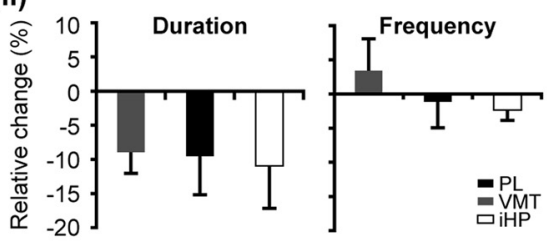

F

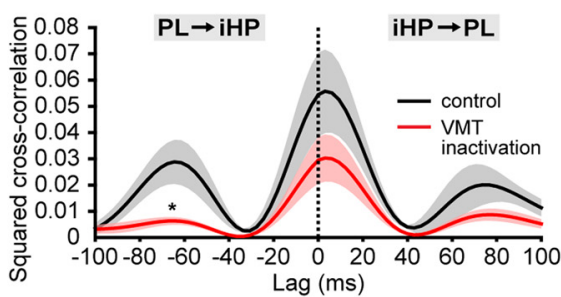

Figure 6. Consequences of transient action potential blockade in either PL or VMT on the patterns of neonatal oscillatory activity in LEC, VMT, and iHP or in PL and iHP, respectively. $A-C$, Effects of temporal PL inactivation by lidocaine on the activity of PL and VMT $(n=5)$ or of PL, iHP, and LEC $(n=6)$. A, Examples of extracellular LFP recordings accompanied by the corresponding color-coded wavelet spectra of the discontinuous oscillatory activity in PL (left), VMT (middle), and iHP (right) of a P9 rat before (control; top), shortly after (middle), and 40 min after lidocaine injection into PL (bottom). Note the partial recovery of oscillatory activity $\sim 40 \mathrm{~min}$ after intracortical injection of lidocaine. $B$, Changes of oscillatory properties after $\mathrm{PL}$ inactivation. Bi, Bar diagrams (mean \pm SEM) illustrating the occurrence of oscillatory events in PL ( $n=11$ pups), VMT ( $n=5$ pups), LEC ( $n=6$ pups), and iHP ( $n=5$ pups) in the presence of lidocaine when normalized to values before lidocaine application (control). Negative values correspond to a decrease, whereas positive values mirror an increase when compared with control. Bii, Relationship between inactivation magnitude in PL and iHP as well as in PL and LEC after lidocaine-induced silencing of neuronal activity in PL. The change in occurrence values of oscillatory bursts in PL is plotted versus the change in occurrence values in iHP (white, $n=5$ rats) or LEC (gray, $n=6$ rats). Biii, Same as Bi for amplitude, duration, and main frequency. C, Average power spectra of oscillatory bursts in PL ( $n=10$ animals) during control (black) and after PL inactivation (red). $\mathbf{D}-\boldsymbol{F}$, Effects of temporal VMT inactivation by lidocaine on the simultaneously recorded activity of VMT, PL, and iHP. $\boldsymbol{D}$, Examples of extracellular LFP recordings accompanied by the corresponding color-coded wavelet spectra of the discontinuous oscillatory activity in VMT (left), PL (middle), and iHP (right) of a P9 rat before (control; top), shortly after (middle), and $40 \mathrm{~min}$ after lidocaine injection into PL (bottom). Note the partial recovery of oscillatory activity $\sim 40$ min after intrathalamic injection of lidocaine. $\boldsymbol{E}$, Changes of oscillatory properties after VMT inactivation. Ei, Bar diagrams (mean \pm SEM) illustrating the occurrence and amplitude of events in VMT, PL, and iHP ( $n=6$ pups) in the presence of lidocaine in VMT when normalized to values before lidocaine application. Negative values correspond to a decrease, whereas positive values mirror an increase when compared with control. Eii, Same as Ei for duration and main frequency. $\boldsymbol{F}$, Squared cross-correlations of the amplitudes of theta-filtered LFP recorded from PL and iHP before (black) and after (red) temporal inactivation of VMT by lidocaine, and averaged for all investigated rats $(n=6)$. Positive time lags of the peaks correspond to iHP leading PL. Short ( $<20 \mathrm{~ms})$ lags of significant peaks correspond to putative monosynaptic interactions, whereas long ( $20-70 \mathrm{~ms}$ ) lags correspond to putative polysynaptic interactions. In $\boldsymbol{C}$ and $\boldsymbol{F}$, the transparent area corresponds to SEM. In $\boldsymbol{A}$ and $\boldsymbol{D}$, red boxes indicate the brain area of lidocaine injection, whereas the green boxes mark the recorded brain regions. 


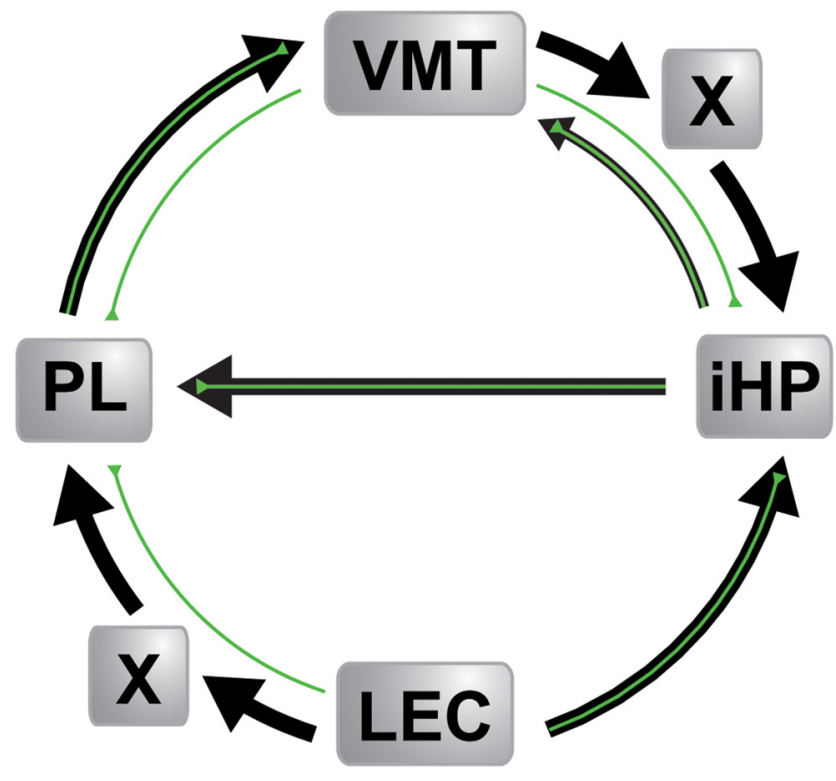

Figure 7. Functional interactions within prelimbic-hippocampal-entorhinal-thalamic networks during neonatal development as resolved by synchrony and causality analysis (black) when accompanied by the distribution of monosynaptic axonal projections (green).

and might contribute to the activation of neonatal PL. This neonatal communication anticipates the adult coupling and may serve to harmonize the development of long-range connectivity with regard to future tasks.

Not only is the ontogeny of network activity within VMT nuclei not understood, but also knowledge about the role of these nuclei in adulthood is rather patchy. VMT nuclei seem to control diverse functions, such as circadian rhythms, reproduction, feeding behavior, and nociception. Moreover, the nuclei of VMT influence mnemonic and executive functions at adulthood (Cassel et al., 2013), and modulate hippocampal spatial processing (Jankowski et al., 2014) by coordinating the activation of PFC and HP (Hembrook et al., 2012; Cholvin et al., 2013). The prefrontal-thalamic-hippocampal circuit has been recently identified as the substrate for goal-directed spatial coding (Ito et al., 2015). The present findings give the first insights into the role of VMT nuclei at neonatal age, suggesting that they enable precise timing of prefrontal-hippocampal networks during the first postnatal week by feedback communication. The relevance of these interactions for the maturation of behavioral abilities relying on the activation of thalamic nuclei within prefrontalhippocampal networks remains to be elucidated.

\section{Physiological significance of entorhinal and thalamic activity for the neonatal development of prefrontal-hippocampal networks}

The communication between adult PFC and HP has been proposed as representing the substrate of decision making and working memory. For example, high coherence between the two brain areas helps to decide which information will be stored in longterm memory (Jones and Wilson, 2005; Benchenane et al., 2010; Spellman et al., 2015).

At a neonatal age, the directed interactions between PFC and $\mathrm{HP}$ are not immediately involved in overt behavior. They seem to be relevant for later cognitive performance at a juvenile age (Krüger et al., 2012) by prestabilizing the neuronal circuitry for future tasks. For this, a precisely tuned coupling within prefrontal-hippocampal networks is mandatory. The pathways identified here that link the prelimbic activity back to hippocampal theta bursts might ensure this early control. This conclusion is supported by (1) the decreased activity in iHP after strong lidocaine-induced silencing of prelimbic firing and (2) the weaker feedback coupling after VMT inactivation, which has been detected by cross-correlation analysis of instantaneous LFP amplitudes at long delays (Fig. $6 F$ ). Moreover, the present data revealed that both putative relay stations, LEC and VMT, are activated during directed prefrontal-hippocampal communication in neonatal rats. However, they seem to have distinct contributions to this early communication.

On the one hand, the neonatal entorhinal theta bursts not only stably couple with the hippocampal oscillatory activity, as previously reported for the adult brain (Hahn et al., 2012), but also drive it, as indicated by amplitude cross-correlation analysis, via embryonically emerging axonal projections from entorhinal layers II/III (Deng et al., 2007). On the other hand, LEC indirectly and weakly couples with PL, despite the early presence of direct entorhinal afferents. Since this direct neonatal connectivity emerges predominantly from clustered neurons in superficial layers II/III and not from neurons in the deep entorhinal layers V/VI, as reported for adults (Insausti et al., 1997), it is likely that the projections from deep entorhinal layers develop later during development, simultaneously with substantial pruning of the projections arising from superficial layers. This process might go in line with the initiation of direct functional coupling between LEC and PL, and the diminishment of neonatal polysynaptic interactions. According to these results, we propose that LEC does not act as a relay station ensuring early feedback communication from PL to iHP, but, similar to its function in the adult brain (Deshmukh et al., 2010; Igarashi et al., 2014), controls and drives, together with other subcortical areas such as medial septum (Brockmann et al., 2011), the theta activity in the hippocampal CA1 area.

In contrast, the nuclei of VMT are in an optimal position for relaying the iHP-driven activity of PL back to iHP. This circuitry loop has been proposed at adulthood to underlie the cognitive tasks involving prefrontal-hippocampal activation (Cassel et al., 2013), such as strategy shifting (Cholvin et al., 2013) and memory generalization (Xu and Südhof, 2013). Recently, directed longrange communication from medial PFC via VMT to HP has been demonstrated to be critical for goal-directed spatial navigation (Ito et al., 2015). Remarkably, the prelimbic afferents reach the thalamic nuclei at the developmental stage of emergence of the hippocampal drive to PL. As confirmed by our previous study (Brockmann et al., 2011) on CA1 lesions, gamma-band activation of local prelimbic networks during NG resulted from the excitatory drive of the hippocampal theta bursts. The prelimbic activation subsequently entrains VMT nuclei via putative monosynaptic projections, which in turn drove the activation of the CA1 area, as shown by the effect of temporal VMT inactivation on hippocampal entrainment. While glutamatergic projections tightly link VMT and HP, the peak of amplitude cross-correlation at a time lag of $\sim 53 \mathrm{~ms}$ suggests a polysynaptic coupling between the two areas. The weaker putative monosynaptic drive from iHP to VMT identified by the directionality analysis suggests that hippocampal activity may equally contribute to the emergence of theta bursts in VMT. However, this contribution seems to be limited, since the thalamic activity strongly decreased after PL inactivation, supporting a critical role of the prelimbic drive for oscillatory entrainment in VMT.

We have previously shown that impairment of neonatal prefrontal-hippocampal communication decreases the performance in rec- 
ognition memory tasks at juvenile age (Krüger et al., 2012). Future studies need to decide whether disturbing the feedback communication via thalamic nuclei is equally detrimental for the maturation of cognitive abilities, and underlies the mnemonic deficits in psychiatric and neurological disorders with developmental time course, such as hypoxic-ischemic encephalopathy (Brockmann et al., 2013; Domnick et al., 2015).

\section{References}

Adhikari A, Sigurdsson T, Topiwala MA, Gordon JA (2010) Cross-correlation of instantaneous amplitudes of field potential oscillations: a straightforward method to estimate the directionality and lag between brain areas. J Neurosci Methods 191:191-200. CrossRef Medline

Benchenane K, Peyrache A, Khamassi M, Tierney PL, Gioanni Y, Battaglia FP, Wiener SI (2010) Coherent theta oscillations and reorganization of spike timing in the hippocampal-prefrontal network upon learning. Neuron 66:921-936. CrossRef Medline

Bitzenhofer SH, Hanganu-Opatz IL (2014) Oscillatory coupling within neonatal prefrontal-hippocampal networks is independent of selective removal of GABAergic neurons in the hippocampus. Neuropharmacology 77:57-67. CrossRef Medline

Bitzenhofer SH, Sieben K, Siebert KD, Spehr M, Hanganu-Opatz IL (2015) Oscillatory activity in developing prefrontal networks results from theta-gamma modulated synaptic inputs. Cell Rep 11:486-497. CrossRef Medline

Bjerknes TL, Langston RF, Kruge IU, Moser EI, Moser MB (2015) Coherence among head direction cells before eye opening in rat pups. Curr Biol 25:103-108. CrossRef Medline

Brockmann MD, Pöschel B, Cichon N, Hanganu-Opatz IL (2011) Coupled oscillations mediate directed interactions between prefrontal cortex and hippocampus of the neonatal rat. Neuron 71:332-347. CrossRef Medline

Brockmann MD, Kukovic M, Schönfeld M, Sedlacik J, Hanganu-Opatz IL (2013) Hypoxia-ischemia disrupts directed interactions within neonatal prefrontal-hippocampal networks. PLoS One 8:e83074. CrossRef Medline

Buetfering C, Allen K, Monyer H (2014) Parvalbumin interneurons provide grid cell-driven recurrent inhibition in the medial entorhinal cortex. Nat Neurosci 17:710-718. CrossRef Medline

Buzsáki G (2010) Neural syntax: cell assemblies, synapsembles, and readers. Neuron 68:362-385. CrossRef Medline

Canto CB, Wouterlood FG, Witter MP (2008) What does the anatomical organization of the entorhinal cortex tell us? Neural Plast 2008:381243. CrossRef Medline

Cassel JC, Pereira de Vasconcelos A, Loureiro M, Cholvin T, Dalrymple-Alford JC, Vertes RP (2013) The reuniens and rhomboid nuclei: neuroanatomy, electrophysiological characteristics and behavioral implications. Prog Neurobiol 111:34-52. CrossRef Medline

Cholvin T, Loureiro M, Cassel R, Cosquer B, Geiger K, De Sa Nogueira D, Raingard H, Robelin L, Kelche C, Pereira de Vasconcelos A, Cassel JC (2013) The ventral midline thalamus contributes to strategy shifting in a memory task requiring both prefrontal cortical and hippocampal functions. J Neurosci 33:8772-8783. CrossRef Medline

Cichon NB, Denker M, Grün S, Hanganu-Opatz IL (2014) Unsupervised classification of neocortical activity patterns in neonatal and pre-juvenile rodents. Front Neural Circuits 8:50. CrossRef Medline

Clement EA, Richard A, Thwaites M, Ailon J, Peters S, Dickson CT (2008) Cyclic and sleep-like spontaneous alternations of brain state under urethane anaesthesia. PLoS One 3:e2004. CrossRef Medline

Colgin LL (2011) Oscillations and hippocampal-prefrontal synchrony. Curr Opin Neurobiol 21:467-474. CrossRef Medline

Couey JJ, Witoelar A, Zhang SJ, Zheng K, Ye J, Dunn B, Czajkowski R, Moser MB, Moser EI, Roudi Y, Witter MP (2013) Recurrent inhibitory circuitry as a mechanism for grid formation. Nat Neurosci 16:318-324. CrossRef Medline

Dawitz J, Kramvis I, Kroon T, JHjorth JJJ, Mansvelder HD, Meredith RM (2012) Synaptic mechanisms underlying early entorhinal network development. Paper presented at 8th FENS, Forum of Neuroscience, Barcelona, Spain, July.

Deng JB, Yu DM, Wu P, Li MS (2007) The tracing study of developing entorhinohippocampal pathway. Int J Dev Neurosci 25:251-258. CrossRef Medline

Deshmukh SS, Yoganarasimha D, Voicu H, Knierim JJ (2010) Theta mod- ulation in the medial and the lateral entorhinal cortices. J Neurophysiol 104:994-1006. CrossRef Medline

Domnick NK, Gretenkord S, De Feo V, Sedlacik J, Brockmann MD, Hanganu-Opatz IL (2015) Neonatal hypoxia-ischemia impairs juvenile recognition memory by disrupting the maturation of prefrontalhippocampal networks. Exp Neurol 273:202-214. CrossRef Medline

Eleore L, López-Ramos JC, Guerra-Narbona R, Delgado-García JM (2011) Role of reuniens nucleus projections to the medial prefrontal cortex and to the hippocampal pyramidal CA1 area in associative learning. PloS One 6:e23538. CrossRef Medline

Fyhn M, Molden S, Witter MP, Moser EI, Moser MB (2004) Spatial representation in the entorhinal cortex. Science 305:1258-1264. CrossRef Medline

Hahn TT, McFarland JM, Berberich S, Sakmann B, Mehta MR (2012) Spontaneous persistent activity in entorhinal cortex modulates corticohippocampal interaction in vivo. Nat Neurosci 15:1531-1538. CrossRef Medline

Hembrook JR, Onos KD, Mair RG (2012) Inactivation of ventral midline thalamus produces selective spatial delayed conditional discrimination impairment in the rat. Hippocampus 22:853-860. CrossRef Medline

Hoover WB, Vertes RP (2007) Anatomical analysis of afferent projections to the medial prefrontal cortex in the rat. Brain Struct Funct 212:149-179. CrossRef Medline

Hoover WB, Vertes RP (2012) Collateral projections from nucleus reuniens of thalamus to hippocampus and medial prefrontal cortex in the rat: a single and double retrograde fluorescent labeling study. Brain Struct Funct 217:191-209. CrossRef Medline

Igarashi KM, Lu L, Colgin LL, Moser MB, Moser EI (2014) Coordination of entorhinal-hippocampal ensemble activity during associative learning. Nature 510:143-147. CrossRef Medline

Insausti R, Herrero MT, Witter MP (1997) Entorhinal cortex of the rat: cytoarchitectonic subdivisions and the origin and distribution of cortical efferents. Hippocampus 7:146-183. Medline

Ito HT, Zhang SJ, Witter MP, Moser EI, Moser MB (2015) A prefrontalthalamo-hippocampal circuit for goal-directed spatial navigation. Nature 522:50-55. CrossRef Medline

Jankowski MM, Islam MN, Wright NF, Vann SD, Erichsen JT, Aggleton JP, O'Mara SM (2014) Nucleus reuniens of the thalamus contains head direction cells. Elife 3:e03075. CrossRef Medline

Jay TM, Witter MP (1991) Distribution of hippocampal CA1 and subicular efferents in the prefrontal cortex of the rat studied by means of anterograde transport of Phaseolus vulgaris-leucoagglutinin. J Comp Neurol 313:574-586. CrossRef Medline

Jerbi K, Lachaux JP, N’Diaye K, Pantazis D, Leahy RM, Garnero L, Baillet S (2007) Coherent neural representation of hand speed in humans revealed by MEG imaging. Proc Natl Acad Sci U S A 104:7676-7681. CrossRef Medline

Jones BF, Witter MP (2007) Cingulate cortex projections to the parahippocampal region and hippocampal formation in the rat. Hippocampus 17:957-976. CrossRef Medline

Jones MW, Wilson MA (2005) Theta rhythms coordinate hippocampal-prefrontal interactions in a spatial memory task. PLoS Biol 3:e402. CrossRef Medline

Kerr KM, Agster KL, Furtak SC, Burwell RD (2007) Functional neuroanatomy of the parahippocampal region: the lateral and medial entorhinal areas. Hippocampus 17:697-708. CrossRef Medline

Knierim JJ, Neunuebel JP, Deshmukh SS (2014) Functional correlates of the lateral and medial entorhinal cortex: objects, path integration and localglobal reference frames. Philos Trans R Soc Lond B Biol Sci 369:20130369. CrossRef Medline

Krüger HS, Brockmann MD, Salamon J, Ittrich H, Hanganu-Opatz IL (2012) Neonatal hippocampal lesion alters the functional maturation of the prefrontal cortex and the early cognitive development in pre-juvenile rats. Neurobiol Learn Mem 97:470 -481. CrossRef Medline

Lachaux JP, Rodriguez E, Martinerie J, Varela FJ (1999) Measuring phase synchrony in brain signals. Hum Brain Mapp 8:194-208. CrossRef Medline

Langston RF, Ainge JA, Couey JJ, Canto CB, Bjerknes TL, Witter MP, Moser EI, Moser MB (2010) Development of the spatial representation system in the rat. Science 328:1576-1580. CrossRef Medline

McKenna JT, Vertes RP (2004) Afferent projections to nucleus reuniens of the thalamus. J Comp Neurol 480:115-142. CrossRef Medline 
Mohns EJ, Blumberg MS (2010) Neocortical activation of the hippocampus during sleep in infant rats. J Neurosci 30:3438-3449. CrossRef Medline

Morales GJ, Ramcharan EJ, Sundararaman N, Morgera SD, Vertes RP (2007) Analysis of the actions of nucleus reuniens and the entorhinal cortex on EEG and evoked population behavior of the hippocampus. Conf Proc IEEE Eng Med Biol Soc 2007:2480-2484. CrossRef Medline

Morrissey MD, Maal-Bared G, Brady S, Takehara-Nishiuchi K (2012) Functional dissociation within the entorhinal cortex for memory retrieval of an association between temporally discontiguous stimuli. J Neurosci 32: 5356-5361. CrossRef Medline

Nicolelis MA, Dimitrov D, Carmena JM, Crist R, Lehew G, Kralik JD, Wise SP (2003) Chronic, multisite, multielectrode recordings in macaque monkeys. Proc Natl Acad Sci U S A 100:11041-11046. CrossRef Medline

Schnitzler A, Gross J (2005) Normal and pathological oscillatory communication in the brain. Nat Rev Neurosci 6:285-296. CrossRef Medline

Sheroziya MG, von Bohlen Und Halbach O, Unsicker K, Egorov AV (2009) Spontaneous bursting activity in the developing entorhinal cortex. J Neurosci 29:12131-12144. CrossRef Medline

Siapas AG, Wilson MA (1998) Coordinated interactions between hippocampal ripples and cortical spindles during slow-wave sleep. Neuron 21:1123-1128. CrossRef Medline

Siapas AG, Lubenov EV, Wilson MA (2005) Prefrontal phase locking to hippocampal theta oscillations. Neuron 46:141-151. CrossRef Medline

Soteropoulos DS, Baker SN (2006) Cortico-cerebellar coherence during a precision grip task in the monkey. J Neurophysiol 95:1194-1206. CrossRef Medline

Spellman T, Rigotti M, Ahmari SE, Fusi S, Gogos JA, Gordon JA (2015) Hippocampal-prefrontal input supports spatial encoding in working memory. Nature 522:309-314. CrossRef Medline

Swanson LW (1981) A direct projection from Ammon's horn to prefrontal cortex in the rat. Brain Res 217:150-154. CrossRef Medline

Takehara-Nishiuchi K, Maal-Bared G, Morrissey MD (2011) Increased entorhinal-prefrontal theta synchronization parallels decreased entorhinal-hippocampal theta synchronization during learning and consol- idation of associative memory. Front Behav Neurosci 5:90. CrossRef Medline

Tan HM, Bassett JP, O’Keefe J, Cacucci F, Wills TJ (2015) The development of the head direction system before eye opening in the rat. Curr Biol 25:479-483. CrossRef Medline

Tehovnik EJ, Sommer MA (1997) Effective spread and timecourse of neural inactivation caused by lidocaine injection in monkey cerebral cortex. J Neurosci Methods 74:17-26. CrossRef Medline

Unichenko P, Yang JW, Luhmann HJ, Kirischuk S (2015) Glutamatergic system controls synchronization of spontaneous neuronal activity in the murine neonatal entorhinal cortex. Pflugers Arch 467:1565-1575. CrossRef Medline

Van Eden CG, Uylings HB (1985) Postnatal volumetric development of the prefrontal cortex in the rat. J Comp Neurol 241:268-274. CrossRef Medline

Varela C, Kumar S, Yang JY, Wilson MA (2014) Anatomical substrates for direct interactions between hippocampus, medial prefrontal cortex, and the thalamic nucleus reuniens. Brain Struct Funct 219:911-929. CrossRef Medline

Vertes RP (2006) Interactions among the medial prefrontal cortex, hippocampus and midline thalamus in emotional and cognitive processing in the rat. Neuroscience 142:1-20. CrossRef Medline

Wills TJ, Cacucci F, Burgess N, O'Keefe J (2010) Development of the hippocampal cognitive map in preweanling rats. Science 328:1573-1576. CrossRef Medline

Wills TJ, Barry C, Cacucci F (2012) The abrupt development of adult-like grid cell firing in the medial entorhinal cortex. Front Neural Circuits 6:21. CrossRef Medline

Xu W, Südhof TC (2013) A neural circuit for memory specificity and generalization. Science 339:1290-1295. CrossRef Medline

Yang JW, Hanganu-Opatz IL, Sun JJ, Luhmann HJ (2009) Three patterns of oscillatory activity differentially synchronize developing neocortical networks in vivo. J Neurosci 29:9011-9025. CrossRef Medline 